\title{
Simulation of a Mathematical Model of Malaria Transmission Dynamics in the Presence of Mosquito Net, Fumigation And Treatment
}

\author{
Titus Ifeanyi Chinebu, Edmund Onwubiko Ezennorom, John U Okwor \\ Department of Computer Science, Madonna University, Elele, Okija, Nigeria
}

\section{ABSTRACT}

We proposed and solved a combined control malaria system of fifteen ordinary differential equations modeling the transmission dynamics of malaria between humans and mosquitoes. Since our aim is to minimize the number of exposed and infectious human, we discussed the disease free equilibrium and estimated the basic reproduction number using the next generation matrix method. The disease free equilibrium was asymptotically stable when the reproduction number is less than one and unstable when it is greater than one. Numerical results are provided using matlab software to confirm the analyzed results. Our findings were that malaria may be controlled using the combined control method, the insecticide treated bed nets, fumigation of the surroundings and active malaria drug as this will reduce the contact rate between human and mosquito through reduction in mosquito population and malaria transmission.

Keyword: Mathematical Model, Next Generation Matrix, Sensitivity Analysis, Malaria, Disease Free Equilibrium, Basic Reproduction Number.

\section{INTRODUCTION}

Malaria disease is one of the serious and sometimes fatal, caused when an infected mosquito carrying the parasite feeds on human. Once that infected mosquito bites a human, the parasite is transmitted and it multiplies in the host liver before infecting and destroying their red blood cells. People who are infected of malaria are usually very sick and show symptoms such as high fever, shaking chills and flulike illness. Anopheles mosquito is the type of mosquito that transmits this parasite. Four kinds of malaria parasites infect humans: Plasmodium falciparum, Plasmodium vivax, Plasmodium ovale and Plasmodium malaria. Some of these parasites cause severe type of malaria than others. They are found in different parts of the world.

Plasmodium falciparum is the type of malaria that its attack usually results in severe infection and if not promptly treated may lead to death. In some places, malaria can be treated and controlled with early diagnosis. However, some countries lack resources to do this effectively.

Prevention of malaria in individuals will generally involve the reduction of human mosquito contact through the use of bed nets, repellents, prospective clothing and mosquito avoidance. This can also include reducing the number of mosquito in houses through the use of screen and insecticides. Wide scale use of mosquito insecticide treated nets has contributed substantially to the fall in malaria morbidity and mortality. In addition to protecting the user, insecticide treated nets protect the community by killing anopheles mosquitoes (Moonen et al, 2010; Godfray, 2013) and should be deployed in all area where malaria is endemic. In as much as this control method is very effective, we should observe that the main mosquito vectors bite outside in the evening or morning and so the protection effect is small.

Indoor residual spraying with insecticide that persist and kill mosquitoes is an important component of malaria control (Bernes et al, 2005; Enayati and Hemingway, 2010; Pluess et al, 2010). World Health Organization (WHO) has developed a global strategy frame work for integrated vector management, which advocates for an evidence - based, integrated approach for vector control and offers guidance for successful operationalisation of different approaches (WHO, 2004; Beier, 2008). 
Control activities at community level can utilize approaches which directly reduces human mosquito contact as well as approaches which reduces the total number of mosquitoes in an area. Such approaches include the reduction in mosquito breeding ground (environmental modification), target the larva stages with chemical or biological agents, and massive insecticide spraying for the adult mosquitoes. Biological control method includes the introduction of fish which eat the mosquito larvae or Bercillus Thuringiensis which excrete larval toxins. Case detection and treatment is another potential control method. Identifying and treating infected persons, especially asymptomatic individuals, will reduce the size of the parasite reservoir within the human population and can lower transmission rate.

\section{Related Literatures}

To improve malaria control, there is need to study and identify the mechanisms by which other sensory cues are detected by mosquito and the potential strategies to block them (Agusto et al, 2013; Buonomo, 2013; Chitnis, 2010; Hawlay, 2003; WHO, 2013, 2014). Insect repellents and other protective odorants can be used to prevent bites. Several studies suggested that people can acquire protection against mosquito bites through regular use of insecticide treated bed net. On the other hand, it is challenging to control malaria using bed nets alone because bed nets are only used for fraction of each day. It is important to examine how to combine bed net usage with other control approaches affects disease spread. To influence mosquito bias requires a combination of disease control strategies, such as fumigation of the surroundings, repellants, and bed nets, but the effectiveness of such a multifaceted approach has not been studied using mathematical models. Mathematical models for transmission dynamics of malaria are useful in providing a better knowledge of the disease, to plan for the future and consider appropriate control measures. Models have played great roles in development of the epidemiology of the disease. The study on malaria using mathematical model originated from works of Ross (1910).According to Ross, if the mosquito population can be reduced to below a certain threshold then malaria can be eradicated. MacDonald (1957) modified the model by including super infection and showed that reducing the number of mosquitoes has little effect on epidemiology of malaria in areas of intense transmission. Dietz and Molinoaux (1974) added two classes of humans in their model and these includes those with low recovery rate (more infectious, greater susceptibility) and those with high recovery rate (less infectious, less susceptibility). Compartmental models of malaria and differential equations are constructed to model the disease (Tumwine et al, 2007; Chiyaka et al, 2007; Chitnis et al, 2006; Yang, 2000; Ngwa and Shu, 2000).

Chitnis et al (2006) performed a bifurcation analysis of malaria model. Malaria transmission model which incorporate immunity in the human population had been studied (Tumwine et al, 2007; Chiyaka et al, 2007; Ngwa and Shu, 2000). Panday et al (2013) developed and analyzed a nonlinear SIS mathematical model for transmission of malaria caused by infected mosquito population on susceptible human population and using chemical to control the disease. They found that their model exhibits three non-negative equilibria which are mosquito and disease free equilibrium $E_{0}$, mosquito persistence and disease free equilibrium $E$ and endemic equilibrium $E^{*}$. El-Nor Osman and Adu (2017) considered SEIR-SEI model of malaria transmission between human and mosquitoes and find that malaria may be controlled by reducing the contact rate between human and mosquito, the use of active malaria drugs, insecticides and mosquito treated nets can also help to reduce mosquitoes population and malaria transmission respectively.

Olayini and Obabiyi (2013) used a system of seven dimensional ordinary differential equations to study the transmission of malaria between humans and mosquitoes with nonlinear forces of infection in form of saturated incidence rates produce antibodies in response to presence of parasite causing malaria in both human and mosquito populations. They investigated the stability analysis of disease free equilibrium and observe that it was asymptotically stable when $R_{0}$ is less than one and unstable when $R_{0}$ is greater than one. Otieno et al (2016) formulated a mathematical model for the dynamics of malaria with four - time dependent control measures in Kenya. These are insecticide treated bed nets, treatment, indoor residual spray and intermittent preventive treatment of malaria in pregnancy. They considered constant control parameters and calculated the reproduction number of disease free and endemic equilibrium, followed by the sensitivity analysis and numerical simulations of the optimal control problems using reasonable parameters. In their study, when $R_{0} \leq 1$, it implies that the disease free equilibrium is globally asymptotically stable, but 
when $R_{0}>1$, it shows that the endemic equilibrium is globally asymptotically stable. Also, at $R_{0}=1$, their model exhibits backward bifurcation and they concluded that control programs that follow these strategies can effectively reduce the spread of malaria disease in different malaria transmission setting in Kenya.

In this paper, we presented the malaria transmission dynamics model using ordinary differential equations made up of fifteen compartments. Here we considered four possibilities in the method which includes the use of insecticide treated mosquito nets, treatment, gain of immunity and fumigation (indoor residual spraying with insecticides, reduction in mosquito breeding ground, and targeting the larva stage s with chemical or biological agents), after which the model is simulate and analyzed.

\section{Model Formulation}

\subsection{THE ASSUMPTIONS OF THE MODEL:}

We assume that treated individuals recover from the infection (Chiyaka, 2013);

$>$ Individuals can acquire protection against mosquito bite through regular use of insecticide treated net (Agusto et al, 2013; Buonomo, 2013; Hawlay et al, 2013);

$>$ Both susceptible, Exposed (Infected), Infectious and Recovered humans die at the same natural rate $\mu_{h}$

$>$ Mosquitoes are infected after biting an infectious human (Olaniyi and Obabiyi, 2013);

$>$ Both susceptible, Exposed (Infected) and Infectious mosquitoes die at the same natural rate $\mu_{h}$

$>$ Infectious human can recover by the effect of the natural immune response (Liehl et al, 2015);

$>$ Treatment may fail due to drug resistance (WHO, 2010; Phyo et al, 2012).

\subsection{The Parameters are defined as follows:}

$\rho_{h} \quad$ Recruitment level of susceptible human;

$\rho_{m} \quad$ Recruitment level of susceptible mosquito;

$\alpha_{h} \quad$ Rate at which susceptible human use mosquito net;

$a\left(1-\alpha_{h}\right) \quad$ Rate at which susceptible human doesn't use mosquito net;

$\beta_{h} \quad$ Rate at which susceptible human use mosquito net and fumigate their surroundings;

$a_{1}\left(1-\beta_{h}\right)$ Rate at which susceptible human who use mosquito net doesn't fumigate their Surroundings; $\gamma_{h} \quad$ Rate at which susceptible human who doesn't use mosquito net fumigate their surroundings;

$a_{2}\left(1-\gamma_{h}\right)$ Rate of susceptible human who doesn't use mosquito net and doesn't fumigate their Surroundings;

$\mu_{h} \quad$ Natural death rate of human;

$\mu_{m} \quad$ Natural death rate of mosquito;

$b, b_{1}, b_{2} \quad$ Biting rate of mosquito;

$\psi_{1 h}, \psi_{2 h}, \psi_{3 h}$ Probability that a bit by an infectious mosquito results in transmission of the disease to Human;

$\psi_{m} \quad$ Probability that a bit results in transmission of the parasite from infectious human to a susceptible mosquito;

$\zeta_{h}$ Rate at which exposed (infected) human move to infectious class;

$\zeta_{m}$ Rate at which exposed (infected) mosquito move to infectious class;

$k \odot$ Rate at which exposed (infected) human obtain treatment;

$k_{1} \quad$ Treatment failure in exposed (infected) human;

$k_{2} \quad$ Treatment failure in infectious human;

$v_{1} J$ Rate at which treated exposed (infected) human move to susceptible class;

$v_{2}$ Rate of exposed (infected) human who recover due to prompt immune response;

$v$ Rate at which recovered human move to susceptible class due to loss of immunity;

$\xi_{h} \quad$ Disease induced death rate of human;

$\xi_{m} 6 /$ Disease induced death rate of mosquito;

$\delta_{h} \quad$ Rate at which infectious human are treated;

$\omega_{h} \quad$ Rate at which treated infectious human move to recovered class;

d Rate at which susceptible human who use mosquito net and fumigate their surroundings move to susceptible class;

$r$ Rate recovery of infectious human due to immune response;

$p_{m} \quad$ Rate at which mosquito die due to fumigation;

The Variables (Compartments) are:

$S_{h} \quad$ Susceptible human;

$S_{m} \quad$ Susceptible mosquito;

$S_{h M N}$ Susceptible human who use mosquito net;

$S_{h W M N}$ Susceptible human who doesn't use mosquito net;

$S_{h M N C}$ Susceptible human who use mosquito net and fumigate their surroundings;

$S_{\text {hWMNC }}$ Susceptible human who doesn't use mosquito net but fumigate their surroundings; 
International Journal of Trend in Scientific Research and Development (IJTSRD) ISSN: 2456-6470

$S_{\text {hMNWC }}$ Susceptible human who use mosquito net $E_{m}$ Exposed (infected) mosquito;

but doesn't fumigate their surroundings; $\quad I_{h} \quad$ Infectious human;

$S_{\text {hWMNCC }} \quad$ Susceptible human who doesn't use $I_{m}$ Infectious mosquito;

mosquito net and doesn't fumigate $I_{h} T$ Infectious but treated human;

their surroundings;

$E_{h} \quad$ Exposed (infected) human;

$E_{h} T \quad$ Exposed (infected) but treated human;

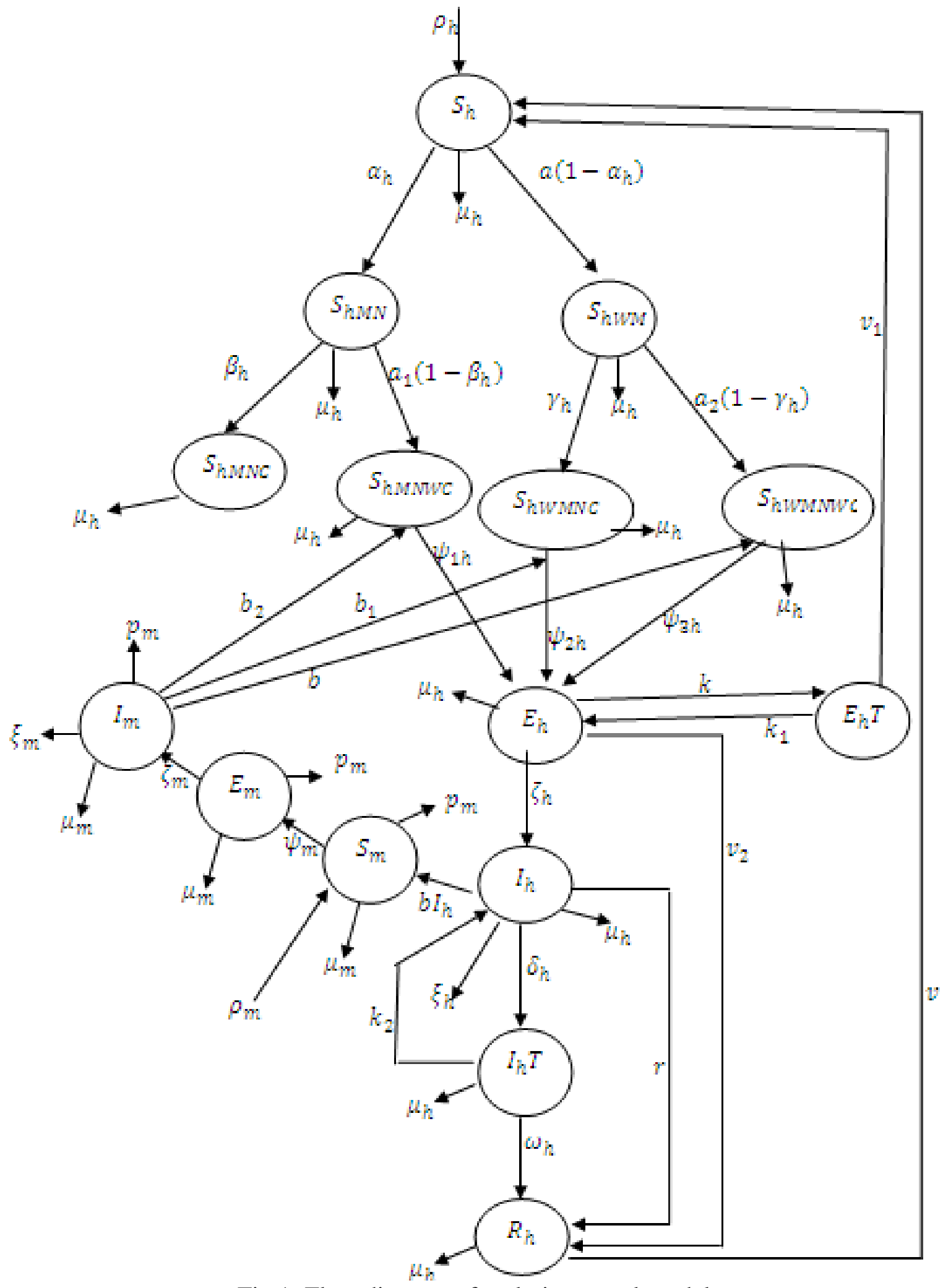

Fig 1: Flow diagram of malaria control model 
We obtain a fifteen dimensional system of ordinary differential equations which describes the progress of the disease and its control as:

$$
\begin{aligned}
& \frac{d S_{h}}{d t}=\rho_{h}+d S_{h M N C}+v_{1} E_{h} T+v R_{h}-\alpha_{h} S_{h}-\mu_{h} S_{h}-a\left(1-\alpha_{h}\right) S_{h} \\
& \frac{d S_{h M N}}{d t}=\alpha S_{h}-\beta_{h} S_{h M N}-a_{1}\left(1-\beta_{h}\right) S_{h M N}-\mu_{h} S_{h M N} \\
& \frac{d S_{h W M N}}{d t}=a\left(1-\alpha_{h}\right) S_{h}-\gamma_{h} S_{h W M N}-a_{2}\left(1-\gamma_{h}\right) S_{h W M N}-\mu_{h} S_{h W M N} \\
& \frac{d S_{h M N C}}{d t}=\beta_{h} S_{h M N}-d S_{h M N C}-\mu_{h} S_{h M N C} \\
& \frac{d S_{h M N W C}}{d t}=a_{1}\left(1-\beta_{h}\right) S_{h M N}+b_{2} I_{m}-\psi_{1 h} S_{h M N W C}-\mu_{h} S_{h M N W C} \\
& \frac{d S_{h W M N C}}{d t}=\gamma_{h} S_{h W M N}+b I_{m}-\psi_{2 h} S_{h W M N C}-\mu_{h} S_{h W M N C} \\
& \frac{d S_{h W M N W C}}{d t}=a_{2}\left(1-\gamma_{h}\right) S_{h W M N}+b_{1} I_{m}-\psi_{3 h} S_{h W M N W C}-\mu_{h} S_{h W M N W C} \\
& \left.\frac{d E_{h}}{d t}=\psi_{1 h} S_{h M N W C}+\psi_{2 h} S_{h W M N C}+\psi_{3 h} S_{h W M N W C}+k_{1} E_{h} T-k E_{h}-v_{2} E_{h}-\zeta_{h} E_{h}-\mu_{h} E_{h}\right\} \\
& \frac{d E_{h} T}{d t}=k E_{h}-v_{1} E_{h} T-\mu_{h} E_{h} T-k_{1} E_{h} T \\
& \frac{d I_{h}}{d t}=\zeta_{h} E_{h}+k_{2} I_{h} T-r I_{h}-\xi_{h} I_{h}-b I_{h}-\delta_{h} I_{h}-\mu_{h} I_{h} \\
& \frac{d I_{h} T}{d t}=\delta_{h} I_{h}-\omega_{h} I_{h} T-\mu_{h} I_{h} T-k_{2} I_{h} T \\
& \frac{d R_{h}}{d t}=\omega_{h} I_{h} T+r I_{h}+v_{2} E_{h}-v R_{h}-\mu_{h} R_{h} \\
& \frac{d S_{m}}{d t}=\rho_{m}+b I_{h}-\psi_{m} S_{m}-p_{m} S_{m}-\mu_{m} S_{m} \\
& \frac{d E_{m}}{d t}=\psi_{m} S_{m}-\zeta_{m} E_{m}-p_{m} E_{m}-\mu_{m} E_{m} \\
& \frac{d I_{m}}{d t}=\zeta_{m} E_{m}-\xi_{m} I_{m}-b_{2} I_{m}-b_{1} I_{m}-b I_{m}-p_{m} I_{m}-\mu_{m} I_{m}
\end{aligned}
$$

From equation (3.1), we observe that the study of the transmission, spread and control of malaria is in two interacting population that human which is the host and mosquito which is the vector. The human and mosquito population size at the time $t$ are respectively denoted as

$$
\begin{gathered}
N_{h}(t)=S_{h}(t)+S_{h M N}(t)+S_{h W M N}(t)+S_{h M N C}(t)+S_{h W M N C}(t)+S_{h M N W C}(t)+ \\
S_{h W M N W C}(t)+E_{h}(t)+E_{h} T(t)+I_{h}(t)+I_{h} T(t)+R_{h}(t) \\
N_{m}(t)=S_{m}(t)+E_{m}(t)+I_{m}(t)
\end{gathered}
$$

\subsection{Existence and Positivity of Solutions}

In equation (3.1), all the parameters are non-negative and we assume stable population with per capita recruitment of susceptible human, susceptible mosquito, death rate of both natural and disease induced and those induced on mosquito due to fumigation. For simplicity, the population size of both human and mosquito are normalized to 1 each that is $S_{h}, S_{h M N}, S_{h W M N}, S_{h M N C}, S_{h W M N C}, S_{h M N W C}, S_{h W M N W C} E_{h}, E_{h} T, I_{h}, I_{h} T, R_{h}$ are respectively the fraction of the Susceptible human, Susceptible human who use mosquito net, Susceptible human who doesn't use mosquito net, Susceptible human who use mosquito net and fumigate their surroundings, Susceptible human who doesn't use mosquito net but fumigate their surroundings, Susceptible human who use mosquito net but doesn't fumigate their surroundings, Susceptible human who doesn't use 
mosquito net and doesn't fumigate their surroundings, Exposed (infected) human, Exposed (infected) but treated human, Infectious human, Infectious but treated human and Recovered human. While $S_{m}, E_{m}, I_{m}$ are respectively fraction of Susceptible mosquito, Exposed (infected) mosquito and Infectious mosquito. Here we shall show that the system is epidemiologically and mathematically well - posed in the feasible region $\Gamma$ given by

Where

$$
\Gamma=H_{h} \times H_{m} \subset \mathbb{R}_{+}^{11} \times \mathbb{R}_{+}^{3}
$$

$$
\begin{gathered}
H_{h}=\left\{\left(S_{h}, S_{h M N}, S_{h W M N}, S_{h M N C}, S_{h W M N C}, S_{h M N W C}, S_{h W M N W C}, E_{h}, E_{h} T, I_{h}, I_{h} T\right) \in \mathbb{R}_{+}^{11}: N_{h} \leq \frac{\rho_{h}}{a+\mu_{h}}\right\} \text { and } \\
H_{m}=\left\{\left(S_{m}, E_{m}, I_{m}\right) \in \mathbb{R}_{+}^{3}: N_{m} \leq \frac{\rho_{m}}{\mu_{m}}\right\} .
\end{gathered}
$$

Theorem 1: There exists a domain $\Gamma$ in which the solution set

$\left\{S_{h}, S_{h M N}, S_{h W M N}, S_{h M N C}, S_{h W M N C}, S_{h M N W C}, S_{h W M N W C}, E_{h}, E_{h} T, I_{h}, I_{h} T, S_{m}, E_{m}, I_{m}\right\}$ is contained and bounded.

Proof: Let the initial conditions be

$$
\begin{gathered}
S_{h}(0)=S_{h 0}, S_{h M N}(0)=S_{h M N 0}, S_{h W M N}(0)=S_{h W M N 0}, S_{h M N C}(0)=S_{h M N C 0}, S_{h W M N C}(0)=S_{h W M N C 0}, \\
S_{h M N W C}(0)=S_{h M N W C 0}, S_{h W M N W C}(0)=S_{h W M N W C 0}, E_{h}(0)=E_{h 0}, E_{h} T(0)=E_{h} T_{0}, I_{h}(0)=I_{h 0}, \\
I_{h} T(0)=I_{h} T_{0}, R_{h}(0)=R_{h 0}, S_{m}(0)=S_{m 0}, E_{m}(0)=E_{m 0}, I_{m}(0)=I_{m 0}
\end{gathered}
$$

Given the solution set $\left\{S_{h}, S_{h M N}, S_{h W M N}, S_{h M N C}, S_{h W M N C}, S_{h M N W C}, S_{h W M N W C}, E_{h}, E_{h} T, I_{h}, I_{h} T, S_{m}, E_{m}, I_{m}\right\}$ with positive initial conditions (3.2), the human population can be represented as

$$
\begin{aligned}
& S_{h}(t)+S_{h M N}(t)+S_{h W M N}(t)+S_{h M N C}(t)+S_{h W M N C}(t)+S_{h M N W C}(t)+S_{h W M N W C}(t) \\
& +E_{h}(t)+E_{h} T(t)+I_{h}(t)+I_{h} T(t)+R_{h}(t)=1
\end{aligned}
$$

Also the mosquito population can be represented thus

$$
S_{m}(t)+E_{m}(t)+I_{m}(t)=1
$$

We can omit the equation for $R_{h}$ in our analysis so that equation (3.1) becomes 
International Journal of Trend in Scientific Research and Development (IJTSRD) ISSN: 2456-6470

$$
\begin{aligned}
& \frac{d S_{h}}{d t}=\rho_{h}+d S_{h M N C}+v_{1} E_{h} T-\alpha_{h} S_{h}-\mu_{h} S_{h}-a\left(1-\alpha_{h}\right) S_{h} \\
& +v\left(1-S_{h}-S_{h M N}-S_{h W M N}-S_{h M N C}-S_{h W M N C}-S_{h M N W C}-S_{h W M N W C}-E_{h}-E_{h} T-I_{h}-I_{h} T\right) \\
& \frac{d S_{h M N}}{d t}=\alpha S_{h}-\beta_{h} S_{h M N}-a_{1}\left(1-\beta_{h}\right) S_{h M N}-\mu_{h} S_{h M N} \\
& \frac{d S_{h W M N}}{d t}=a\left(1-\alpha_{h}\right) S_{h}-\gamma_{h} S_{h W M N}-a_{2}\left(1-\gamma_{h}\right) S_{h W M N}-\mu_{h} S_{h W M N} \\
& \frac{d S_{h M N C}}{d t}=\beta_{h} S_{h M N}-d S_{h M N C}-\mu_{h} S_{h M N C} \\
& \frac{d S_{h M N W C}}{d t}=a_{1}\left(1-\beta_{h}\right) S_{h M N}+b_{2} I_{m}-\psi_{1 h} S_{h M N W C}-\mu_{h} S_{h M N W C} \\
& \frac{d S_{h W M N C}}{d t}=\gamma_{h} S_{h W M N}+b I_{m}-\psi_{2 h} S_{h W M N C}-\mu_{h} S_{h W M N C} \\
& \frac{d S_{h W M N W C}}{d t}=a_{2}\left(1-\gamma_{h}\right) S_{h W M N}+b_{1} I_{m}-\psi_{3 h} S_{h W M N W C}-\mu_{h} S_{h W M N W C} \\
& \frac{d E_{h}}{d t}=\psi_{1 h} S_{h M N W C}+\psi_{2 h} S_{h W M N C}+\psi_{3 h} S_{h W M N W C}+k_{1} E_{h} T-k E_{h}-v_{2} E_{h}-\zeta_{h} E_{h}-\mu_{h} E_{h} \\
& \frac{d E_{h} T}{d t}=k E_{h}-v_{1} E_{h} T-\mu_{h} E_{h} T-k_{1} E_{h} T \\
& \frac{d I_{h}}{d t}=\zeta_{h} E_{h}+k_{2} I_{h} T-r I_{h}-\xi_{h} I_{h}-b I_{h}-\delta_{h} I_{h}-\mu_{h} I_{h} \\
& \frac{d I_{h} T}{d t}=\delta_{h} I_{h}-\omega_{h} I_{h} T-\mu_{h} I_{h} T-k_{2} I_{h} T \\
& \frac{d R_{h}}{d t}=\omega_{h} I_{h} T+r I_{h}+v_{2} E_{h}-v R_{h}-\mu_{h} R_{h} \\
& \frac{d S_{m}}{d t}=\rho_{m}+b I_{h}-\psi_{m} S_{m}-p_{m} S_{m}-\mu_{m} S_{m} \\
& \frac{d E_{m}}{d t}=\psi_{m} S_{m}-\zeta_{m} E_{m}-p_{m} E_{m}-\mu_{m} E_{m} \\
& \frac{d I_{m}}{d t}=\zeta_{m} E_{m}-\xi_{m} I_{m}-b_{2} I_{m}-b_{1} I_{m}-b I_{m}-p_{m} I_{m}-\mu_{m} I_{m}
\end{aligned}
$$

Then the time derivatives of $H_{h}(t) a n d H_{m}(t)$ along solutions of system (3.1) for humans and mosquitoes respectively are denoted by

$$
\begin{gathered}
H_{h}(t)=S_{h}(t)+S_{h M N}(t)+S_{h W M N}(t)+S_{h M N C}(t)+S_{h W M N C}(t)+S_{h M N W C}(t)+S_{h W M N W C}(t) \\
+E_{h}(t)+E_{h} T(t)+I_{h}(t)+I_{h} T(t) \\
H_{h}(t)=\rho_{h}+d S_{h M N C}+v_{1} E_{h} T-\alpha_{h} S_{h}-\mu_{h} S_{h}-a\left(1-\alpha_{h}\right) S_{h} \\
+v\left(1-S_{h}-S_{h M N}-S_{h W M N}-S_{h M N C}-S_{h W M N C}-S_{h M N W C}-S_{h W M N W C}-E_{h}-E_{h} T-I_{h}-I_{h} T\right) \\
+\alpha S_{h}-\beta_{h} S_{h M N}-a_{1}\left(1-\beta_{h}\right) S_{h M N}-\mu_{h} S_{h M N}+a\left(1-\alpha_{h}\right) S_{h}-\gamma_{h} S_{h W M N}-a_{2}\left(1-\gamma_{h}\right) S_{h W M N} \\
-\mu_{h} S_{h W M N}+\beta_{h} S_{h M N}-d S_{h M N C}-\mu_{h} S_{h M N C}+a_{1}\left(1-\beta_{h}\right) S_{h M N}+b_{2} I_{m}-\psi_{1 h} S_{h M N W C}-\mu_{h} S_{h M N W C} \\
+\gamma_{h} S_{h W M N}+b I_{m}-\psi_{2 h} S_{h W M N C}-\mu_{h} S_{h W M N C}+a_{2}\left(1-\gamma_{h}\right) S_{h W M N}+b_{1} I_{m}-\psi_{3 h} S_{h W M N W C} \\
-\mu_{h} S_{h W M N W C}+\psi_{1 h} S_{h M N W C}+\psi_{2 h} S_{h W M N C}+\psi_{3 h} S_{h W M N W C}+k_{1} E_{h} T-k E_{h}-v_{2} E_{h}-\zeta_{h} E_{h}-\mu_{h} E_{h} \\
+k E_{h}-\mu_{h} I_{h} T-v_{1} E_{h} T-k_{1} E_{h} T+\zeta_{h} E_{h}+k_{2} I_{h} T-\mu_{h} I_{h}-r I_{h}-\xi_{h} I_{h}-b I_{h}-\delta_{h} I_{h}+\delta_{h} I_{h}-\omega_{h} I_{h} T \\
-\mu_{h} I_{h} T-k_{2} I_{h} T
\end{gathered}
$$

Where $S_{h}+S_{h M N}+S_{h W M N}+S_{h M N C}+S_{h W M N C}+S_{h M N W C}+S_{h W M N W C}+E_{h}+E_{h} T+I_{h}+I_{h} T=H_{h}$ It is worthy to note that in the absence of the disease $b I_{m}, b_{1} I_{m}, b_{2} I_{m}, v_{2} E_{h}, r I_{h}, \xi_{h} I_{h}, b I_{h}$ and $\omega_{h} I_{h} T$ will all be zero. Therefore we have 
International Journal of Trend in Scientific Research and Development (IJTSRD) ISSN: 2456-6470

$$
\begin{gathered}
H_{h}(t)=\rho_{h}-\left(\mu_{h} S_{h}+\mu_{h} S_{h M N}+\mu_{h} S_{h W M N}+\mu_{h} S_{h M N C}+\mu_{h} S_{h W M N C}+\mu_{h} S_{h M N W C}+\mu_{h} S_{h W M N W C}+\mu_{h} E_{h}\right. \\
\left.+\mu_{h} E_{h} T+\mu_{h} I_{h}+\mu_{h} I_{h} T\right)+v\left(1-H_{h}\right) \\
H_{h}(t)=\rho_{h}-\mu_{h} H_{h}+v\left(1-H_{h}\right) \\
H_{h}(t)=\rho_{h}+v-\left(\mu_{h}+v\right) H_{h} \\
H_{h}(t)+\left(\mu_{h}+v\right) H_{h} \leq \rho_{h}+v
\end{gathered}
$$

Integrating both sides of equation (3.7) using integrating factor method (Kar and Jana 2013; Birkhoff and Roffa, 1989; Chinebu et al, 2018) we have

$$
\begin{gathered}
H_{h}^{\prime}+p(t) d t=f(t) \\
H_{h} \leq e^{-\int p(t) d t}\left(\int e^{\int p(t) d t} f(t) d t+c\right)
\end{gathered}
$$

Where

We then have the integrating factor as

$$
p(t)=\mu_{h}+\operatorname{vand} f(t)=\rho_{h}+v
$$

$$
r(t)=e^{\int p(t) d t}=e^{\int\left(\mu_{h}+v\right) d t}=e^{\left(\mu_{h}+v\right) t}
$$

Now integrating (3.7) using the value of the integrating factor $r(t)=e^{\left(\mu_{h}+v\right) t}$ we have

$$
\begin{gathered}
H_{h}(t) \leq \frac{1}{r(t)}\left[\int r(t) \cdot f(t) d t+c\right] \\
H_{h}(t) \leq \frac{1}{e^{\left(\mu_{h}+v\right) t}}\left[\int e^{\left(\mu_{h}+v\right) t} \cdot\left(\rho_{h}+v\right) d t+c\right] \\
\leq \frac{1}{e^{\left(\mu_{h}+v\right) t}}\left[\left(\rho_{h}+v\right) \int e^{\left(\mu_{h}+v\right) t} \cdot d t+c\right] \\
\leq \frac{1}{e^{\left(\mu_{h}+v\right) t}}\left[\frac{\left(\rho_{h}+v\right)}{\left(\mu_{h}+v\right)} e^{\left(\mu_{h}+v\right) t}+c\right] \\
H_{h}(t) \leq \frac{\rho_{h}+v}{\mu_{h}+v}+c e^{-\left(\mu_{h}+v\right) t} \text { Sientific }
\end{gathered}
$$

Where $\mathrm{c}$ is the constant of integration and if we let $t \rightarrow \infty$ we have

But

$$
H_{h}(t)=\frac{\rho_{h}+v}{\mu_{h}+v}
$$

$$
=S_{h}+S_{h M N}+S_{h W M N}+S_{h M N C}+S_{h W M N C}+S_{h M N W C}+S_{h W M N W C}+E_{h}+E_{h} T+I_{h}+I_{h} T
$$

$$
S_{h} \leq \frac{\rho_{h}\left(\beta_{h}+d_{2}+\mu_{h}\right)\left(d+\mu_{h}\right)}{\left(\mu_{h}+\alpha_{h}+d_{1}\right)\left(\beta_{h}+d_{2}+\mu_{h}\right)\left(d+\mu_{h}\right)-d \beta_{h} \alpha_{h}}
$$

which at disease free equilibrium gives

Also,

$$
S_{h}=\frac{\rho_{h}}{a+\mu_{h}}
$$

$$
\begin{gathered}
H_{m}(t)=S_{m}(t)+E_{m}(t)+I_{m}(t) \\
H_{m}(t)=\rho_{m}+b I_{h}-\psi_{m} S_{m}-p_{m} S_{m}-\mu_{m} S_{m}+\psi_{m} S_{m}-\zeta_{m} E_{m}-p_{m} E_{m}-\mu_{m} E_{m} \\
+\zeta_{m} E_{m}-\xi_{m} I_{m}-b_{2} I_{m}-b_{1} I_{m}-b I_{m}-p_{m} I_{m}-\mu_{m} I_{m}
\end{gathered}
$$

But in the absence of the disease, $b_{2} I_{m}, b_{1} I_{m}, b I_{m}, \xi_{m} I_{m}, p_{m} S_{m}, p_{m} E_{m}, p_{m} I_{m}$ will all be zero and we obtain

$$
H_{m}(t)=\rho_{m}-\mu_{m}\left(S_{m}+E_{m}+I_{m}\right)
$$

If we let $S_{m}+E_{m}+I_{m}=H_{m}$, then we have

Where

$$
\begin{gathered}
H_{m}(t)=\rho_{m}-\mu_{h} H_{m} \\
H_{m}(t)+\mu_{m} H_{m} \leq \rho_{h} \quad H_{m}^{\prime}+p(t) d t=f(t) \\
H_{m} \leq e^{-\int p(t) d t}\left(\int e^{\int p(t) d t} f(t) d t+c_{1}\right)
\end{gathered}
$$

$$
p(t)=\mu_{m} \text { and } f(t)=\rho_{m}
$$


We then have the integrating factor as

$$
r(t)=e^{\int p(t) d t}=e^{\int \mu_{m} d t}=e^{\left(\mu_{m}\right) t}
$$

Now integrating (3.11) using the value of the integrating factor $r(t)=e^{\left(\mu_{m}\right) t}$ we have

$$
\begin{gathered}
H_{m}(t) \leq \frac{1}{r(t)}\left[\int r(t) \cdot f(t) d t+c_{1}\right] \\
H_{m}(t) \leq \frac{1}{e^{\left(\mu_{m}\right) t}}\left[\int e^{\left(\mu_{m}\right) t} \cdot\left(\rho_{m}\right) d t+c_{1}\right] \\
\leq \frac{1}{e^{\left(\mu_{m}\right) t}}\left[\left(\rho_{m}\right) \int e^{\left(\mu_{m}\right) t} \cdot d t+c_{1}\right] \\
\leq \frac{1}{e^{\left(\mu_{m}\right) t}}\left[\frac{\rho_{m}}{\mu_{m}} e^{\left(\mu_{m}\right) t}+c_{1}\right] \\
H_{m}(t) \leq \frac{\rho_{m}}{\mu_{m}}+c_{1} e^{-\left(\mu_{m}\right) t}
\end{gathered}
$$

Where $c_{1}$ is the constant of integration and if we let $t \rightarrow \infty$ we have

But

$$
H_{m}(t)=\frac{\rho_{m}}{\mu_{m}}=S_{m}+E_{m}+I_{m}
$$

$$
S_{m}=\frac{\rho_{m}}{\mu_{m}+\psi_{m}+p_{m}}
$$

which reduces to $\frac{\rho_{m}}{\mu_{m}}$ at disease free equilibrium. We observe from the dynamics described by thee system (3.1), (3.9) and (3.13) that the region

$$
\begin{gathered}
\Gamma=\left\{\left(S_{h}, S_{h M N}, S_{h W M N}, S_{h M N C}, S_{h W M N C}, S_{h M N W C}, S_{h W M N W C}, E_{h}, E_{h} T, I_{h}, I_{h} T, S_{m}, E_{m}, I_{m}\right) \in \mathbb{R}_{+}^{14}: N_{h}\right. \\
\left.\leq \frac{\rho_{h}}{a+\mu_{h}}: N_{m} \leq \frac{\rho_{m}}{\mu_{m}}\right\} \text { Trend in Scientific }
\end{gathered}
$$

is positive invariant. This simply implied that the system for humans and mosquitoes are respectively well posed mathematically. Then for the initial stating point $H_{h} \subset \mathbb{R}_{+}^{11}$ and $H_{m} \subset \mathbb{R}_{+}^{3}$, the trajectory lies in $\Gamma$. Thus, we focus our attention only on the region $\Gamma$.

\subsection{Stability of the Disease Free Equilibrium (DFE)}

The disease free equilibrium state denotes the point at which the differential equations of system (3.1) are equal to zero and it refers to absence of infection. Therefore, all the infected class will be zero and the entire population for human will comprise of Susceptible human, Susceptible human who use mosquito net, Susceptible human who doesn't use mosquito net, Susceptible human who use mosquito net and fumigate their surroundings, Susceptible human who doesn't use mosquito net but fumigate their surroundings, Susceptible human who use mosquito net but doesn't fumigate their surroundings, Susceptible human who doesn't use mosquito net and doesn't fumigate their surroundings while the entire population for mosquito will be the susceptible mosquito only.

At equilibrium state we set the rate of change of each variable to be equal to zero, that is

$$
\begin{gathered}
\frac{d S_{h}}{d t}=\frac{d S_{h M N}}{d t}=\frac{d S_{h W M N}}{d t}=\frac{d S_{h M N C}}{d t}=\frac{d S_{h M N W C}}{d t}=\frac{d S_{h W M N C}}{d t}=\frac{d S_{h W M N W C}}{d t}=\frac{d E_{h}}{d t}=\frac{d E_{h} T}{d t}=\frac{d I_{h}}{d t}= \\
\frac{d I_{h}}{d t}=\frac{d I_{h} T}{d t}=\frac{d R_{h}}{d t}=0
\end{gathered}
$$

Let $\left(S_{h}, S_{h M N}, S_{h W M N}, S_{h M N C}, S_{h W M N C}, S_{h M N W C}, S_{h W M N W C}, E_{h}, E_{h} T, I_{h}, I_{h} T, S_{m}, E_{m}, I_{m}\right)$

$$
=\left(S_{h}{ }^{*}, S_{h M N}{ }^{*}, S_{h W M N}{ }^{*}, S_{h M N C}{ }^{*}, S_{h W M N C}{ }^{*}, S_{h M N W C}{ }^{*}, S_{h W M N W C}{ }^{*}, E_{h}{ }^{*}, E_{h} T^{*}, I_{h}{ }^{*}, I_{h} T^{*}, S_{m}{ }^{*}, E_{m}{ }^{*}, I_{m}{ }^{*}\right)
$$

at equilibrium state. If we substitute $E_{h}{ }^{*}=E_{h} T^{*}=I_{h}{ }^{*}=I_{h} T^{*}=E_{m}{ }^{*}=I_{m}{ }^{*}=0$ into equation (3.1) we have the disease free equilibrium state for human and mosquito calculated as 
International Journal of Trend in Scientific Research and Development (IJTSRD) ISSN: 2456-6470

$$
\left.\begin{array}{c}
\rho_{h}+d S_{h M N C}+v_{1} E_{h} T+v R_{h}-\alpha_{h} S_{h}-\mu_{h} S_{h}-a\left(1-\alpha_{h}\right) S_{h}=0 \\
\alpha S_{h}-\beta_{h} S_{h M N}-a_{1}\left(1-\beta_{h}\right) S_{h M N}-\mu_{h} S_{h M N}=0 \\
a\left(1-\alpha_{h}\right) S_{h}-\gamma_{h} S_{h W M N}-a_{2}\left(1-\gamma_{h}\right) S_{h W M N}-\mu_{h} S_{h W M N}=0 \\
\beta_{h} S_{h M N}-d S_{h M N C}-\mu_{h} S_{h M N C}=0 \\
a_{1}\left(1-\beta_{h}\right) S_{h M N}+b_{2} I_{m}-\psi_{1 h} S_{h M N W C}-\mu_{h} S_{h M N W C}=0 \\
\gamma_{h} S_{h W M N}+b I_{m}-\psi_{2 h} S_{h W M N C}-\mu_{h} S_{h W M N C}=0 \\
a_{2}\left(1-\gamma_{h}\right) S_{h W M N}+b_{1} I_{m}-\psi_{3 h} S_{h W M N W C}-\mu_{h} S_{h W M N W C}=0 \\
\psi_{1 h} S_{h M N W C}+\psi_{2 h} S_{h W M N C}+\psi_{3 h} S_{h W M N C C}+k_{1} E_{h} T-k E_{h}-v_{2} E_{h}-\zeta_{h} E_{h}-\mu_{h} E_{h}=0 \\
k E_{h}-v_{1} E_{h} T-\mu_{h} E_{h} T-k_{1} E_{h} T=0 \\
\zeta_{h} E_{h}+k_{2} I_{h} T-r_{h}-\xi_{h} I_{h}-b I_{h}-\delta_{h} I_{h}-\mu_{h} I_{h}=0 \\
\delta_{h} I_{h}-\omega_{h} I_{h} T-\mu_{h} I_{h} T-k_{2} I_{h} T=0 \\
\omega_{h} I_{h} T+r I_{h}+v_{2} E_{h}-v R_{h}-\mu_{h} R_{h}=0 \\
\rho_{m}+b I_{h}-\psi_{m} S_{m}-p_{m} S_{m}-\mu_{m} S_{m}=0 \\
\psi_{m} S_{m}-\zeta_{m} E_{m}-p_{m} E_{m}-\mu_{m} E_{m}=0 \\
\zeta_{m} E_{m}-\xi_{m} I_{m}-b_{2} I_{m}-b_{1} I_{m}-b I_{m}-p_{m} I_{m}-\mu_{m} I_{m}=0
\end{array}\right\}
$$

If we let $a\left(1-\alpha_{h}\right)=d_{1}, a_{1}\left(1-\beta_{h}\right)=d_{2}, a_{2}\left(1-\gamma_{h}\right)=d_{3}$, and label equation (3.15) as itoxv, we calculate the equilibrium point for both human and mosquito.

Substituting $E_{h}{ }^{*}=E_{h} T^{*}=I_{h}{ }^{*}=I_{h} T^{*}=E_{m}{ }^{*}=I_{m}{ }^{*}=0$ we have from (3.15xii) as

But $I_{h} T=I_{h}=E_{h}=0$

$$
\omega_{h} I_{h} T+r I_{h}+v_{2} E_{h}-v R_{h}-\mu_{h} R_{h}=0
$$

$$
\left(v+\mu_{h}\right) R_{h}=0 \quad \Rightarrow R_{h}=0
$$

From (3.15iv) we have

Equation (3.15ii) gives

$$
\begin{gathered}
\beta_{h} S_{h M N}-d S_{h M N C}-\mu_{h} S_{h M N C}=0 \\
S_{h M N C}=\frac{\beta_{h} S_{h M N}}{d+\mu_{h}} \text { Research and } \\
\alpha_{h} S_{h}-\beta_{h} S_{h M N}-a_{1}\left(1-\beta_{h}\right) S_{h M N}-\mu_{h} S_{h M N}=0 \\
S_{h M N}=\frac{\alpha_{h} S_{h}}{\beta_{h}+d_{2}+\mu_{h}} 2456-6470
\end{gathered}
$$

Substituting (3.18) into (3.17) we obtain

$$
S_{h M N C}=\frac{\beta_{h} \alpha_{h} S_{h}}{\left(d+\mu_{h}\right)\left(\beta_{h}+d_{2}+\mu_{h}\right)}
$$

Substituting (3.16) and (3.19) into (3.15i) gives

$$
\begin{gathered}
\rho_{h}+\frac{d \beta_{h} \alpha_{h} S_{h}}{\left(d+\mu_{h}\right)\left(\beta_{h}+d_{2}+\mu_{h}\right)}-\left(\alpha_{h}+d_{1}+\mu_{h}\right) S_{h}=0 \\
\rho_{h}\left(d+\mu_{h}\right)\left(\beta_{h}+d_{2}+\mu_{h}\right)+\left[d \beta_{h} \alpha_{h}-\left(\alpha+d_{1}+\mu_{h}\right)\left(d+\mu_{h}\right)\left(\beta_{h}+d_{2}+\mu_{h}\right)\right] S_{h}=0
\end{gathered}
$$

Substituting (3.20) into (3.18) gives

$$
S_{h M N}=\frac{\alpha_{h} \rho_{h}\left(d+\mu_{h}\right)}{\left(\alpha_{h}+d_{1}+\mu_{h}\right)\left(d+\mu_{h}\right)\left(\beta_{h}+d_{2}+\mu_{h}\right)-d \beta_{h} \alpha_{h}}
$$

From (3.15iii) we have

$$
S_{h W M N}=\frac{a\left(1-\alpha_{h}\right) S_{h}-\gamma_{h} S_{h W M N}-a_{2}\left(1-\gamma_{h}\right) S_{h W M N}-\mu_{h} S_{h W M N}=0}{d_{1} \rho_{h}\left(d+\mu_{h}\right)\left(\beta_{h}+d_{2}+\mu_{h}\right)}
$$

Substituting (3.21) into (3.17) gives

$$
S_{h M N C}=\frac{\beta_{h} \alpha_{h} \rho_{h}}{\left(\alpha_{h}+d_{1}+\mu_{h}\right)\left(d+\mu_{h}\right)\left(\beta_{h}+d_{2}+\mu_{h}\right)-d \beta_{h} \alpha_{h}}
$$


From equation $(3.15 v)$ we have

$$
S_{h M N W C}=\frac{a_{1}\left(1-\beta_{h}\right) S_{h M N}+b_{2} I_{m}-\psi_{1 h} S_{h M N W C}-\mu_{h} S_{h M N W C}=0}{\left(\psi_{1 h}+\mu_{h}\right)\left[\left(\alpha_{h}+d_{1}+\mu_{h}\right)\left(d+\mu_{h}\right)\left(\beta_{h}+d_{2}+\mu_{h}\right)-d \beta_{h} \alpha_{h}\right]}
$$

From equation (3.15vi) we have

$$
S_{h W M N C}=\frac{\gamma_{h} S_{h W M N}+b I_{m}-\psi_{2 h} S_{h W M N C}-\mu_{h} S_{h W M N C}=0}{\left(\psi_{2 h}+\mu_{h}\right)\left[\left(\gamma_{h}+d_{3}+\mu_{h}\right)\left[\left(\alpha_{h}+d_{1}+\mu_{h}\right)\left(d+\mu_{h}\right)\left(\beta_{h}+d_{2}+\mu_{h}\right)-d \beta_{h} \alpha_{h}\right]\right]}
$$

From equation (3.15vii) we have

$$
S_{h W M N W C}=\frac{a_{2}\left(1-\gamma_{h}\right) S_{h W M N}+b_{1} I_{m}-\psi_{3 h} S_{h W M N W C}-\mu_{h} S_{h W M N W C}=0}{\left(\psi_{3 h}+\mu_{h}\right)\left[\left(\gamma_{h}+d_{3}+\mu_{h}\right)\left[\left(\alpha_{h}+d_{1}+\mu_{h}\right)\left(d+\mu_{h}\right)\left(\beta_{h}+d_{2}+\mu_{h}\right)-d \beta_{h} \alpha_{h}\right]\right]}
$$

From equation (15xiii) we have

$$
S_{m}=\frac{\rho_{m}}{\mu_{m}} \rho_{m}+b I_{h}-\psi_{m} S_{m}-p_{m} S_{m}-\mu_{m} S_{m}=0
$$

\subsection{Analysis of the System of Control}

We analyzed the equilibrium point and the basic reproduction number of the systems having in mind that the control parameters $\alpha_{h}(t), \beta_{h}(t), \gamma_{h}(t), \delta_{h}(t), k(t)$ and $p(t)$ are constant functions.

\subsection{Equilibrium Point and Basic Reproduction Number $\boldsymbol{R}_{o}$}

Here we calculate the disease free equilibrium point of system (3.1) which shows that the population is free of malaria infection and this implies that there isn't need for control. Therefore, the control parameters will be equal to zero.

\subsection{Disease Free Equilibrium}

Let $M_{0}{ }^{*}$ be the disease free equilibrium point for both human and mosquito, then we have

Remember that

$$
M_{0}{ }^{*}=\left(S_{h 0}, S_{h M N 0}, S_{h W M N 0}, S_{h M N C 0}, S_{h W M N C 0}, S_{h M N W C 0}, S_{h W M N W C 0}, 0,0,0,0,0, S_{m 0}, 0,0\right)
$$

and

$$
S_{h 0}=\frac{\rho_{h}\left(d+\mu_{h}\right)\left(\beta_{h}+d_{2}+\mu_{h}\right)}{\left(\alpha+d_{1}+\mu_{h}\right)\left(d+\mu_{h}\right)\left(\beta_{h}+d_{2}+\mu_{h}\right)-d \beta_{h} \alpha_{h}}
$$

$$
\begin{aligned}
& a\left(1-\alpha_{h}\right)=d_{1}, a_{1}\left(1-\beta_{h}\right)=d_{2}, a_{2}\left(1-\gamma_{h}\right)=d_{3}, \\
& \therefore S_{h 0}=\frac{\rho_{h}}{a+\mu_{h}} \\
& S_{\text {hMN }}=\frac{\alpha_{h} \rho_{h}\left(d+\mu_{h}\right)}{\left(\alpha_{h}+d_{1}+\mu_{h}\right)\left(d+\mu_{h}\right)\left(\beta_{h}+d_{2}+\mu_{h}\right)-d \beta_{h} \alpha_{h}}=0 \\
& S_{h W M N 0}=\frac{d_{1} \rho_{h}\left(d+\mu_{h}\right)\left(\beta_{h}+d_{2}+\mu_{h}\right)}{\left(\gamma_{h}+d_{3}+\mu_{h}\right)\left[\left(\alpha_{h}+d_{1}+\mu_{h}\right)\left(d+\mu_{h}\right)\left(\beta_{h}+d_{2}+\mu_{h}\right)-d \beta_{h} \alpha_{h}\right]} \\
& S_{\text {hWMNO }}=\frac{a \rho_{h}}{\left(a+\mu_{h}\right)\left(a_{2}+\mu_{h}\right)} \\
& S_{h M N C 0}=\frac{\beta_{h} \alpha_{h} \rho_{h}}{\left(\alpha_{h}+d_{1}+\mu_{h}\right)\left(d+\mu_{h}\right)\left(\beta_{h}+d_{2}+\mu_{h}\right)-d \beta_{h} \alpha_{h}}=0 \\
& S_{\text {hWMNCO }}=\frac{\gamma_{h} d_{1} \rho_{h}\left(d+\mu_{h}\right)\left(\beta_{h}+d_{2}+\mu_{h}\right)}{\left(\psi_{2 h}+\mu_{h}\right)\left[\left(\gamma_{h}+d_{3}+\mu_{h}\right)\left[\left(\alpha_{h}+d_{1}+\mu_{h}\right)\left(d+\mu_{h}\right)\left(\beta_{h}+d_{2}+\mu_{h}\right)-d \beta_{h} \alpha_{h}\right]\right]}=0 \\
& S_{\text {hMNWC0 }}=\frac{d_{2} \alpha_{h} \rho_{h}\left(d+\mu_{h}\right)}{\left(\psi_{1 h}+\mu_{h}\right)\left[\left(\alpha_{h}+d_{1}+\mu_{h}\right)\left(d+\mu_{h}\right)\left(\beta_{h}+d_{2}+\mu_{h}\right)-d \beta_{h} \alpha_{h}\right]}=0 \\
& S_{h W M N W C 0}=\frac{d_{3} d_{1} \rho_{h}\left(d+\mu_{h}\right)\left(\beta_{h}+d_{2}+\mu_{h}\right)}{\left(\psi_{3 h}+\mu_{h}\right)\left[\left(\gamma_{h}+d_{3}+\mu_{h}\right)\left[\left(\alpha_{h}+d_{1}+\mu_{h}\right)\left(d+\mu_{h}\right)\left(\beta_{h}+d_{2}+\mu_{h}\right)-d \beta_{h} \alpha_{h}\right]\right]} \\
& =\frac{a a_{2} \rho_{h}}{\mu_{h}\left(a+\mu_{h}\right)\left(a_{2}+\mu_{h}\right)}
\end{aligned}
$$


The disease free equilibrium point for human and mosquito is

$$
S_{m 0}=S_{m}=\frac{\rho_{m}}{\mu_{m}+\psi_{m}}=\frac{\rho_{m}}{\mu_{m}}
$$

$$
M_{0}^{*}=\left(\frac{\rho_{h}}{a+\mu_{h}}, 0, \frac{a \rho_{h}}{\left(a+\mu_{h}\right)\left(a_{2}+\mu_{h}\right)}, 0,0,0, \frac{a a_{2} \rho_{h}}{\mu_{h}\left(a+\mu_{h}\right)\left(a_{2}+\mu_{h}\right)}, 0,0,0,0,0, \frac{\rho_{m}}{\mu_{m}}, 0,0\right)
$$

\subsection{Basic Reproduction Number}

The basic Reproduction number of epidemiological model usually denoted by $R_{0}$ is the average number of secondary infections caused by a single infectious individual during their entire infectious lifetime (Driessche and Watmough, 2002). It helps to show whether the infection will be endemic or not i.e., spread through the population or not. When the basic reproduction number is less than one $\left(R_{0}<1\right)$, the disease free equilibrium point is locally asymptotically stable while when it is greater than one $\left(R_{0}>1\right)$, the disease free equilibrium point is unstable.To obtain $R_{0}$ for model system (1) we use the next generation matrix technique (Van den Driessche and Watmough, 2002; Diekmann et al 1990). We shall start with those equations of the model that describe the production of new infections and change in state among infected humans and mosquitoes.

Let $Q=\left[S_{h}, S_{h M N}, S_{h W M N}, S_{h M N C}, S_{h W M N C}, S_{h M N W C}, S_{h W M N W C}, E_{h}, E_{h} T, I_{h}, I_{h} T, S_{m}, E_{m}, I_{m}\right]^{T} \quad$ where $\quad T$ denote transpose. Then

where

$$
\frac{d Q}{d t}=F(Q)-V(Q)
$$

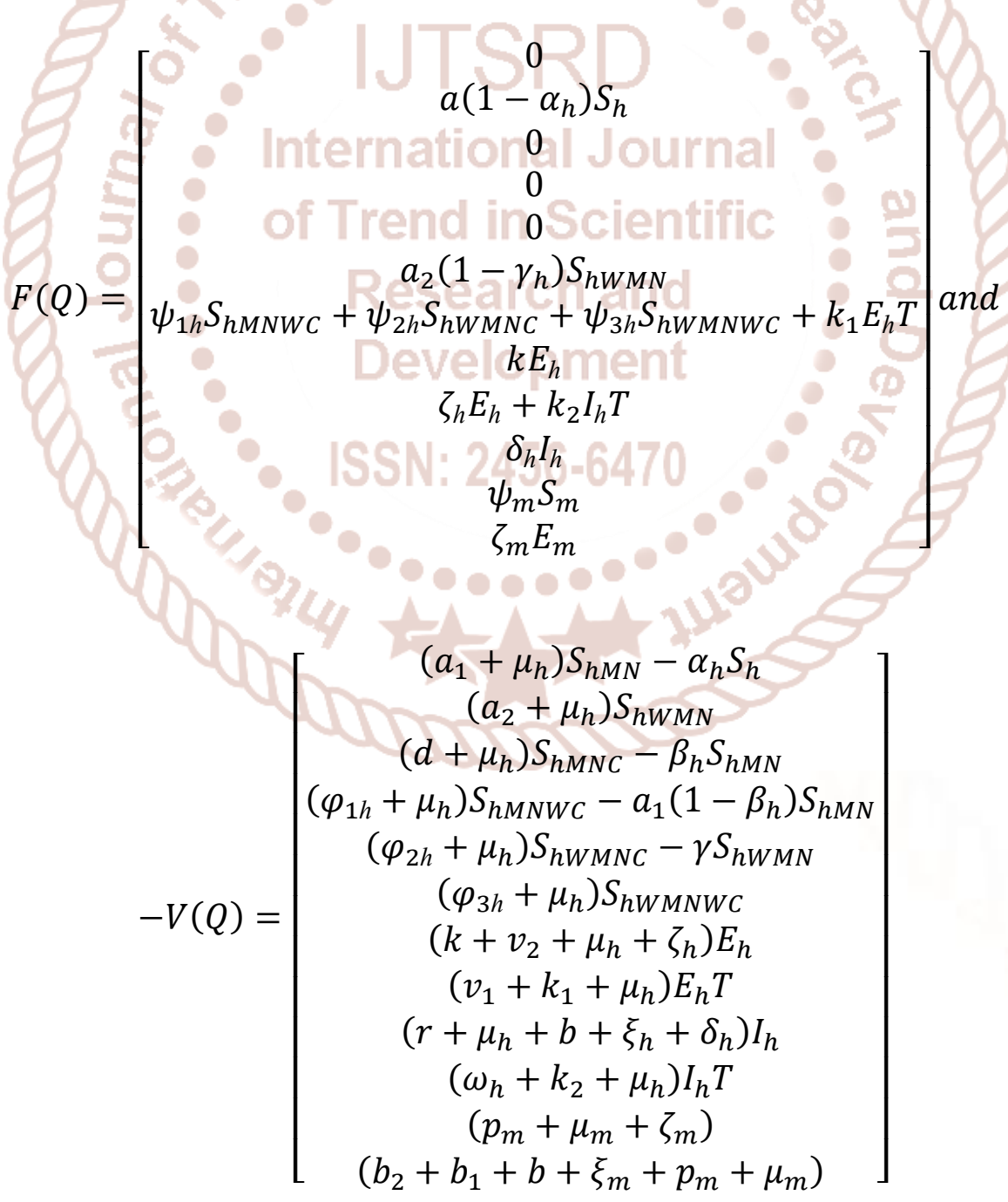


If we find the derivatives of $F$ and $V$ at the disease free equilibrium point $M_{0}$ we obtain $F$ and $V$ respectively as;

$\mathrm{F}:=\left[\begin{array}{cccccccccccc}0 & 0 & 0 & 0 & 0 & 0 & 0 & 0 & 0 & 0 & 0 & 0 \\ 0 & 0 & 0 & 0 & 0 & 0 & 0 & 0 & 0 & 0 & 0 & 0 \\ 0 & 0 & 0 & 0 & 0 & 0 & 0 & 0 & 0 & 0 & 0 & 0 \\ 0 & 0 & 0 & 0 & 0 & 0 & 0 & 0 & 0 & 0 & 0 & \mathrm{~b}_{2} \\ 0 & 0 & 0 & 0 & 0 & 0 & 0 & 0 & 0 & 0 & 0 & \mathrm{~b}_{1} \\ 0 & \mathrm{a}_{2}\left(1-\gamma_{\mathrm{h}}\right) & 0 & 0 & 0 & 0 & 0 & 0 & 0 & 0 & 0 & \mathrm{~b} \\ 0 & 0 & 0 & \psi_{1 \mathrm{~h}} & \psi_{2 \mathrm{~h}} & \psi_{3 \mathrm{~h}} & \mathrm{k}_{1} & 0 & 0 & 0 & 0 & 0 \\ 0 & 0 & 0 & 0 & 0 & 0 & \mathrm{k} & 0 & 0 & 0 & 0 & 0 \\ 0 & 0 & 0 & 0 & 0 & 0 & \zeta_{\mathrm{h}} & 0 & \mathrm{k}_{2} & 0 & 0 & 0 \\ 0 & 0 & 0 & 0 & 0 & 0 & 0 & 0 & \delta_{\mathrm{h}} & 0 & 0 & 0 \\ 0 & 0 & 0 & 0 & 0 & 0 & 0 & 0 & 0 & 0 & 0 & 0 \\ 0 & 0 & 0 & 0 & 0 & 0 & 0 & 0 & 0 & 0 & \zeta_{\mathrm{m}} & 0\end{array}\right]$

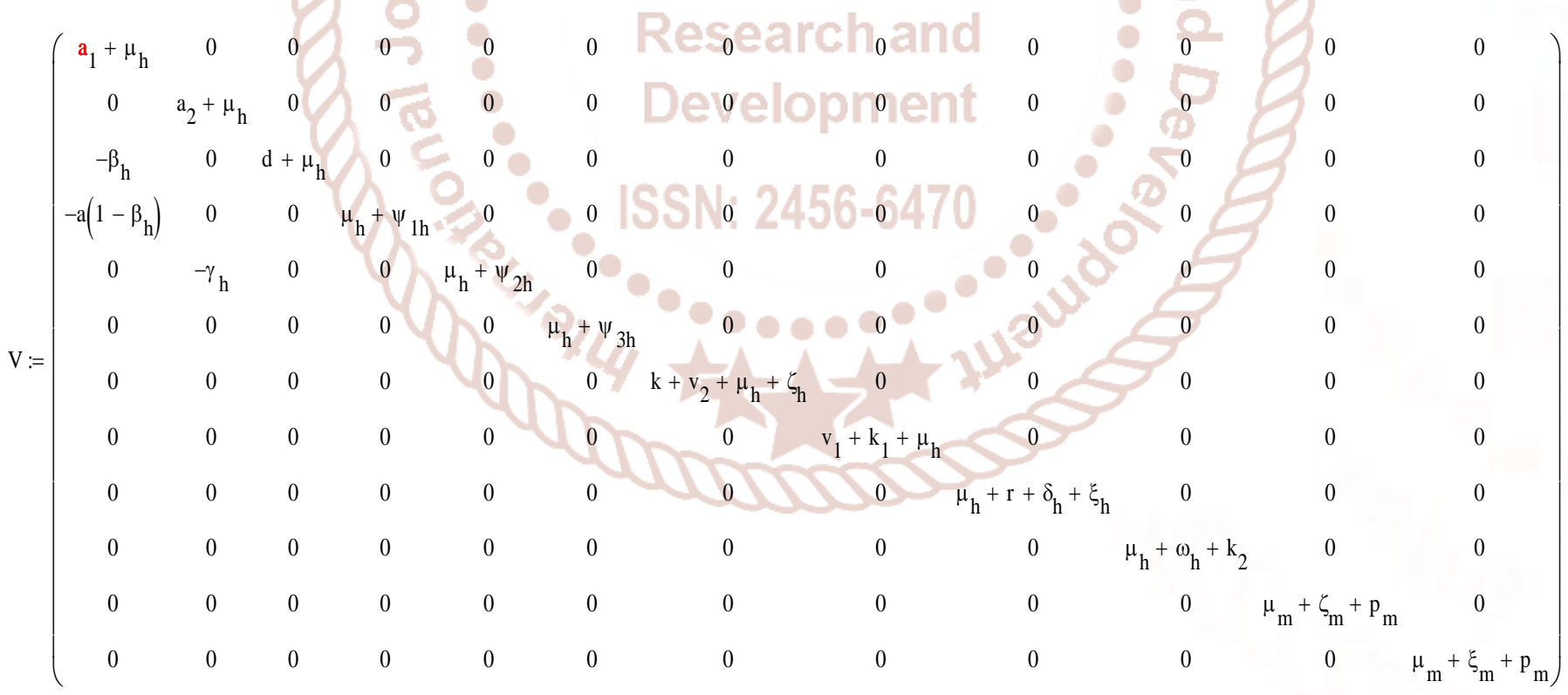




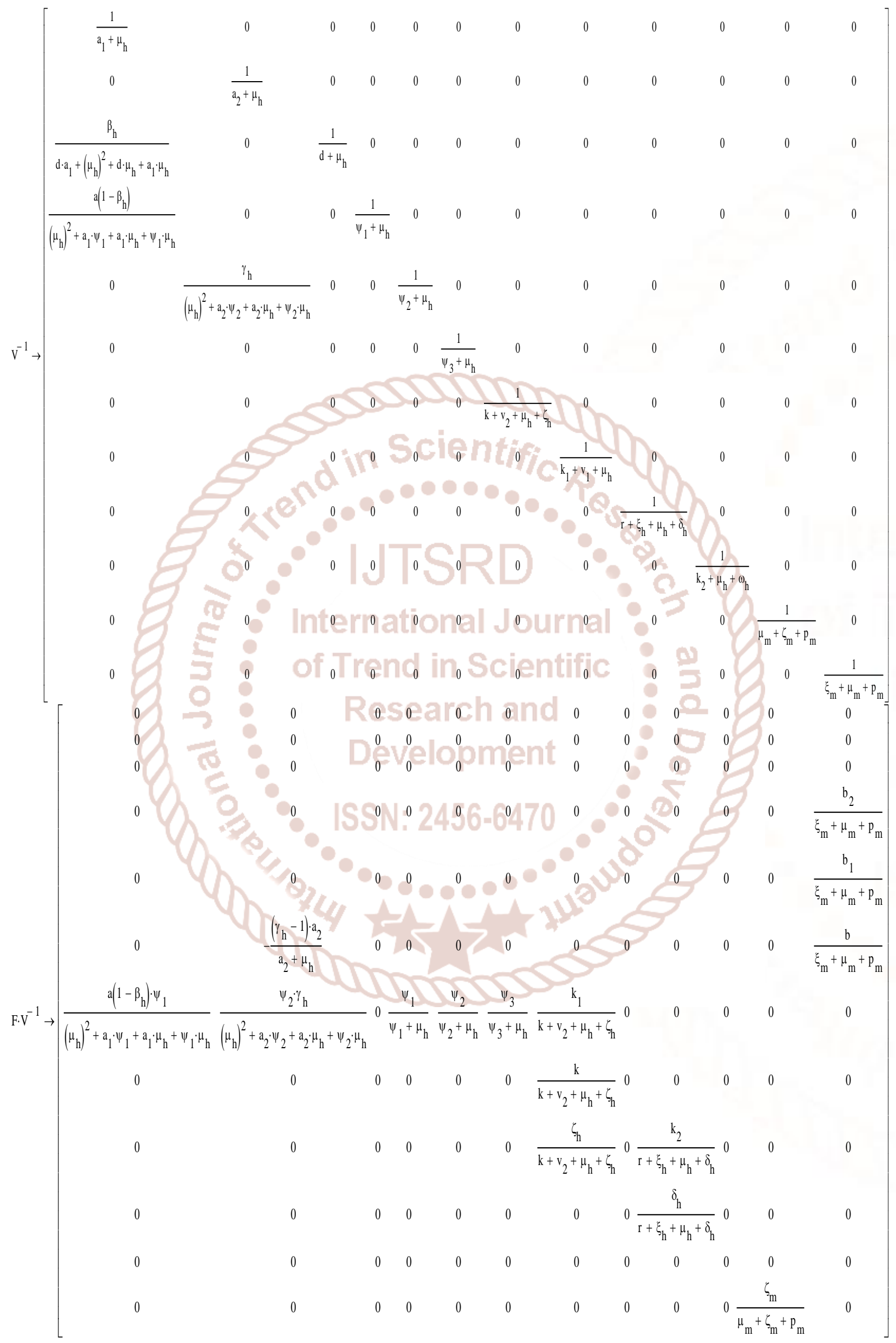


Observe from determinant of matrices that

$>$ When every element in a row (or column) of an $m \times m$ (square) matrix is zero, the value of the determinant will be zero.

Since the matrix above satisfies this property, we state that the

$$
\left|F . V^{-1}\right|=0
$$

To obtain the basic reproduction number $R_{0}$ we use the trace and determinant of the matrix and we let $F V^{-1}=$ A.

Let

$$
R_{0}=\varphi(A)=\frac{1}{2}\left(\operatorname{trace}(A)+\sqrt{\operatorname{trace}(A)^{2}-4 \operatorname{det}(A)}\right)
$$

Since $\operatorname{det}(A)=0$, we have that

$$
R_{0}=\frac{k_{1}}{k+v_{2}+\mu_{h}+\zeta_{h}}+\frac{k_{2}}{r+\mu_{h}+\xi_{h}+\delta_{h}}
$$

Therefore, if $R_{0}<1$, then the disease free equilibrium is stable and malaria infection can be controlled using the three control measure. On the other hand, if $R_{0}>1$, then the disease free equilibrium is unstable and the malaria infection will persists' in the population.

\subsection{Sensitivity Analysis of the Basic Reproduction Number $\boldsymbol{R}_{0}$ of Malaria with Control}

We conducted sensitivity analysis to determine the nature of the model. $R_{0}$ is the expected number of new infected humans as a result of introducing one infected human in a population at a disease free equilibrium in the absence of disease control methods. If $R_{0}<1$, each infected human infect on average less than one individual in the population and hence the disease dies out. But if $R_{0}>1$, each infected human infects more than one individual in the population and thus the disease is able to invade the susceptible population. In other words, $R_{0}$ is used here to determine the effectiveness of the control measure. To calculate the value of $R_{0}$ we

\begin{tabular}{|c|c|c|}
\hline Parameters & & $\begin{array}{l}\text { Value } \\
\text { Range }\end{array}$ \\
\hline$\rho_{h}$ & Recruitment level of susceptible human & 0.0814 \\
\hline$\rho_{m}$ & Recruitment level of susceptible mosquito & 0.0184 \\
\hline$\alpha_{h}$ & Rate at which susceptible human use mosquito net & 0.75 \\
\hline$a\left(1-\alpha_{h}\right)$ & Rate at which susceptible human doesn't use mosquito net & 0.25 \\
\hline$\beta_{h}$ & $\begin{array}{c}\text { Rate at which susceptible human use mosquito net and fumigate their } \\
\text { surroundings }\end{array}$ & 0.1125 \\
\hline$\gamma_{h}$ & $\begin{array}{l}\text { Rate at which susceptible human who doesn't use mosquito net fumigate their } \\
\text { surroundings }\end{array}$ & 0.11 \\
\hline$\mu_{h}$ & Natural death rate of human & 0.0000548 \\
\hline$\mu_{m}$ & Natural death rate of mosquito & 0.67 \\
\hline \multicolumn{3}{|c|}{ (l) } \\
\hline$b_{h 1,2,3}$ & Biting rate of mosquito & 0.12 \\
\hline$\psi_{h 1,2,3}$ & $\begin{array}{l}\text { Probability that a bit by an infectious mosquito results in transmission of the } \\
\text { disease to Human }\end{array}$ & 0.0200 \\
\hline$\psi_{m}$ & $\begin{array}{l}\text { Probability that a bit results in transmission of the parasite from infectious human } \\
\text { to a susceptible mosquito }\end{array}$ & $\begin{array}{l}0.5 \text { or } \\
0.8333\end{array}$ \\
\hline$\zeta_{h}$ & Rate at which exposed (infected) human move to infectious class & 0.0588 \\
\hline$\zeta_{m}$ & Rate at which exposed (infected) mosquito move to infectious class & 0.0017 \\
\hline$k$ & Rate at which exposed (infected) human obtain treatment & 0.28 \\
\hline
\end{tabular}
used the parameter values in table 1 .

Table 1 Parameter values for the calculation of $R_{0}$ and Numerical Simulation of the Model. 
International Journal of Trend in Scientific Research and Development (IJTSRD) ISSN: 2456-6470

\begin{tabular}{|c|c|c|}
\hline$k_{1}$ & Treatment failure in exposed (infected) human & 0.09 \\
\hline$k_{2}$ & Treatment failure in infectious human & 0.017 \\
\hline$v_{1}$ & Rate at which treated exposed (infected) human move to susceptible class & 0.05 \\
\hline$v_{2}$ & Rate of exposed (infected) human who recover due to prompt immune response & 0.00137 \\
\hline$v$ & Rate at which recovered human move to susceptible class due to loss of immunity & 0.000137 \\
\hline$\xi_{h}$ & Disease induced death rate of human & 0.02326 \\
\hline$\xi_{m}$ & Disease induced death rate of mosquito & 0.07 \\
\hline$\delta_{h}$ & Rate at which infectious human are treated & 0.3325 \\
\hline$\omega_{h}$ & Rate at which treated infectious human move to recovered class & 0.000548 \\
\hline$d$ & $\begin{array}{l}\text { Rate at which susceptible human who use mosquito net and fumigate their } \\
\text { surroundings move to susceptible class. }\end{array}$ & 0.1 \\
\hline$r$ & Rate recovery of infectious human due to immune response & 0.0035 \\
\hline$p_{m}$ & Rate at which mosquito die due to fumigation & 0.15 \\
\hline$a_{1}\left(1-\beta_{h}\right)$ & $\begin{array}{c}\text { Rate at which susceptible human who use mosquito net doesn't fumigate their } \\
\text { surroundings }\end{array}$ & 0.8875 \\
\hline$a_{2}\left(1-\gamma_{h}\right)$ & $\begin{array}{c}\text { Rate of susceptible human who doesn't use mosquito net and doesn't fumigate } \\
\text { their surroundings }\end{array}$ & 0.89 \\
\hline
\end{tabular}

To calculate the value of $R_{0}$, we use the parameters as stated in table.......

$$
\begin{gathered}
R_{0}=\frac{k_{1}}{k+v_{2}+\mu_{h}+\zeta_{h}}+\frac{k_{2}}{r+\mu_{h}+\xi_{h}+\delta_{h}} \\
R_{0}=\frac{0.0175}{0.28+0.00137+0.0000548+0.0588}+\frac{0.09}{0.0035+0.0000548+0.02326+0.3325} \\
=\frac{0.0175}{0.3402248}+\frac{0.3593148}{0.2645309807+0.0487038107} \\
R_{0}=0.3132347914<1
\end{gathered}
$$

For the effect of $k_{1}$ on $R_{0}$ we have

For the effect of $k$ on $R_{0}$ we have

$$
\mathrm{E}_{k_{1}}^{R_{0}}=\frac{\partial R_{0}}{\partial k_{1}} \times \frac{R_{0}}{k_{1}}=0.7280723238
$$

For the effect of $v_{2}$ on $R_{0}$ we have

$$
\mathrm{E}_{k}^{R_{0}}=\frac{\partial R_{0}}{\partial k} \times \frac{R_{0}}{k}=-0.8698062743
$$

For the effect of $\zeta_{h}$ on $R_{0}$ we have

$$
\mathrm{E}_{v_{2}}^{R_{0}}=\frac{\partial R_{0}}{\partial v_{2}} \times \frac{R_{0}}{v_{2}}=-177.77061763
$$

For the effect of $r$ on $R_{0}$ we have

$$
\mathrm{E}_{\zeta_{h}}^{R_{0}}=\frac{\partial R_{0}}{\partial \zeta_{h}} \times \frac{R_{0}}{\zeta_{h}}=-4.1419346281
$$

For the effect of $k_{2}$ on $R_{0}$ we have

$$
\mathrm{E}_{r}^{R_{0}}=\frac{\partial R_{0}}{\partial r} \times \frac{R_{0}}{r}=-12.130810762
$$

For the effect of $\mu_{h}$ on $R_{0}$ we have

$$
\mathrm{E}_{k_{2}}^{R_{0}}=\frac{\partial R_{0}}{\partial k_{2}} \times \frac{R_{0}}{k_{2}}=49.814621989
$$

$$
\mathrm{E}_{\mu_{h}}^{R_{0}}=\frac{\partial R_{0}}{\partial \mu_{h}} \times \frac{R_{0}}{\mu_{h}}=-5219.0436284
$$

For the effect of $\xi_{h}$ on $R_{0}$ we have 
For the effect of $\delta_{h}$ on $R_{0}$ we have

$$
\mathrm{E}_{\xi_{h}}^{R_{0}}=\frac{\partial R_{0}}{\partial \xi_{h}} \times \frac{R_{0}}{\xi_{h}}=-1.8253583204
$$

$$
\mathrm{E}_{\delta_{h}}^{R_{0}}=\frac{\partial R_{0}}{\partial \delta_{h}} \times \frac{R_{0}}{\delta_{h}}=-0.1276927339
$$

\section{Table 2: The Effect of the Parameters on $\boldsymbol{R}_{\mathbf{0}}$}

\begin{tabular}{|c|c|}
\hline Parameters & Effect on $\boldsymbol{R}_{0}$ \\
\hline$k_{1}$ & 0.7280723238 \\
\hline$k$ & -0.8698062743 \\
\hline$v_{2}$ & -177.77061763 \\
\hline$\zeta_{h}$ & -4.1419346281 \\
\hline$r$ & -12.130810762 \\
\hline$k_{2}$ & 49.814621989 \\
\hline$\mu_{h}$ & -5219.0436284 \\
\hline$\xi_{h}$ & -1.8253583204 \\
\hline$\delta_{h}$ & -0.1276927339 \\
\hline
\end{tabular}

The sensitivity of $\mathrm{E}\left(\mathrm{k} \_1\right)$ and $\mathrm{E}\left(\mathrm{k} \_2\right)$ are positive and this implies that the value of $\mathrm{R} \_0$ increases as the values of $\mathrm{k} \_1$ and $\mathrm{k} \_(2)$ increases. Contrary to this, the values of the parameters $\mathrm{E}(\mathrm{k}), \mathrm{E}\left(\delta_{-} \mathrm{h}\right), \mathrm{E}\left(\xi_{-} \mathrm{h}\right), \mathrm{E}\left(\mu_{-} \mathrm{h}\right), \mathrm{E}(\mathrm{r}), \mathrm{E}\left(\zeta_{-} \mathrm{h}\right)$ and $\mathrm{E}(\mathrm{v})$ are negative, indicating that the value of $\mathrm{R} \_0$ decreases as $\mathrm{k}, \delta_{-} \mathrm{h}, \xi_{-} \mathrm{h}, \mu_{-} \mathrm{h}, \mathrm{r}, \zeta_{-} \mathrm{h}$ and $\mathrm{v}$ increases. This simply shows that these parameters contribute in reducing that rate of transmission of malaria infection over time in the population. If these parameters are maintained, the transmission of the disease may decrease causing the cases of malaria infection in the population to reduce and go below the endemicity threshold.

\section{Results and Discussion}

In this section, we study numerically the behavior of system (3.1) using the parameter values given in table 1 and by considering initial conditions, $\Pi=\left\{S_{h}(0), S_{h M N}(0), S_{h W M N}(0), S_{h M N C}(0), S_{h W M N C}(0)\right.$,

$\left.S_{h M N W C}(0), S_{h W M N W C}(0), E_{h}(0), E_{h} T(0), I_{h}(0) I_{h} T(0), R_{h}(0), S_{m}(0), E_{m}(0), I_{m}(0)\right\}$. The numerical simulation are conducted using Matlab software and the results are given in figure $2-4$ where figure $2 \mathrm{a}-2 \mathrm{~b}$ illustrate the behavior of the reproductive number for different values of the model parameter $k$ and $r$ respectively. Figures $3 \mathrm{i}-3 \mathrm{xv}$ and $4 \mathrm{i}-4 \mathrm{xv}$ shows the varying effects of when there is no control and when there is control respectively.

The basic reproduction number of the system is given by

$$
R_{0}=\frac{k_{1}}{k+v_{2}+\mu_{h}+\zeta_{h}}+\frac{k_{2}}{r+\mu_{h}+\xi_{h}+\delta_{h}}=0.3132347914<1
$$

This simply shows that the basic reproduction number is less than one, hence the disease free equilibrium is stable which indicates that the malaria infection can be controlled in the population using the control measures stated in the model. However, it also confirms the result of the sensitivity analysis of $R_{0}$ in table 2 and we then state that with effective use of insecticide treated bed nets, modification of the environment through fumigation and treatment of infectious human, the future number of malaria infection cases will reduce in the population.

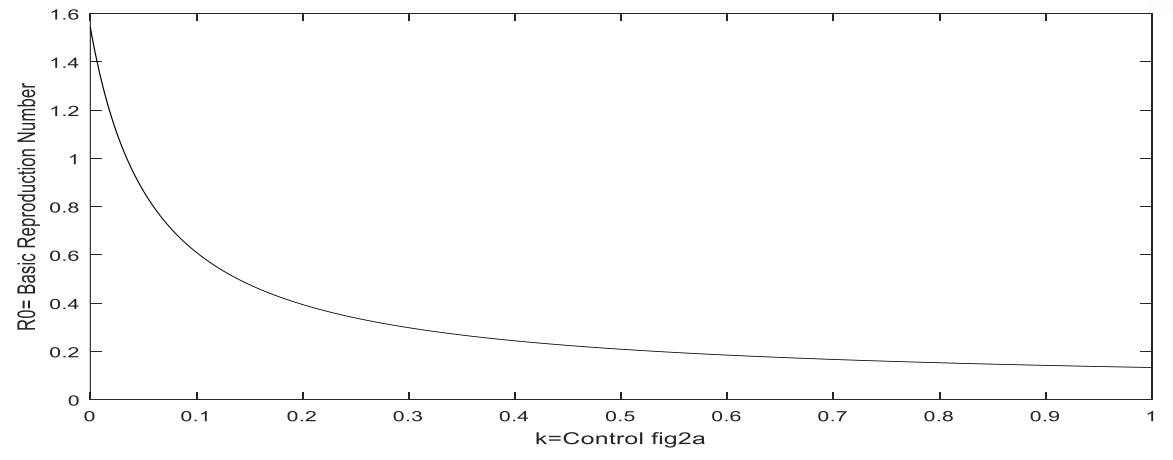




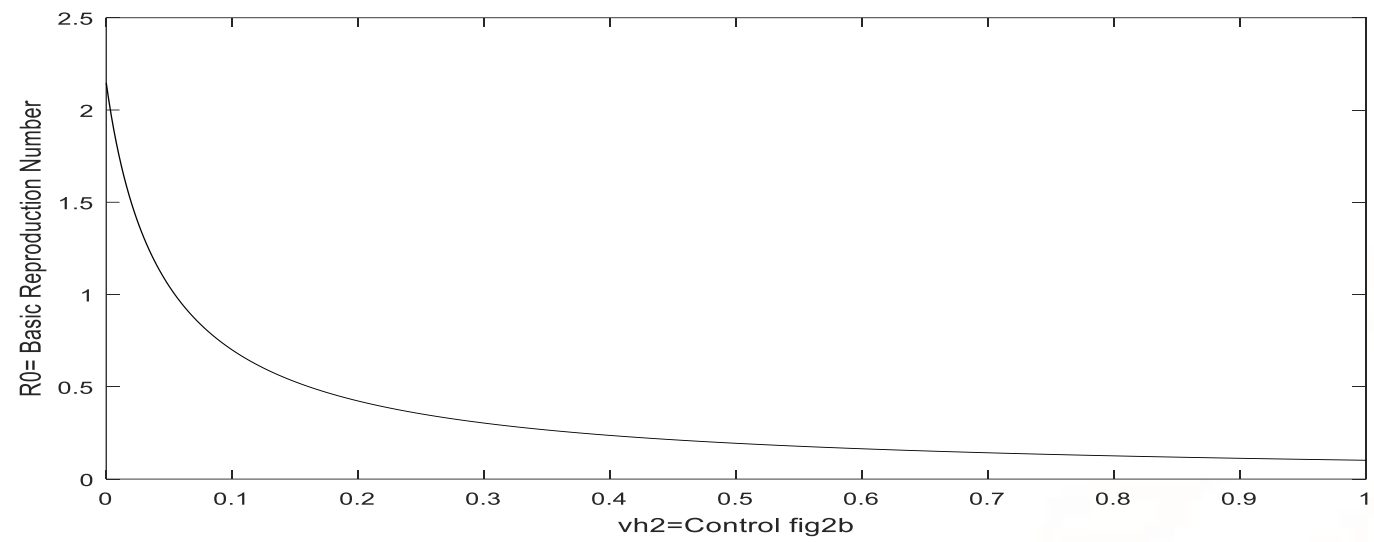

Figures $(2 \mathrm{a}-2 \mathrm{~b})$ : Numerical Simulation of the basic Reproduction Number $R_{0}$ using different rate of $k$ and $r$.

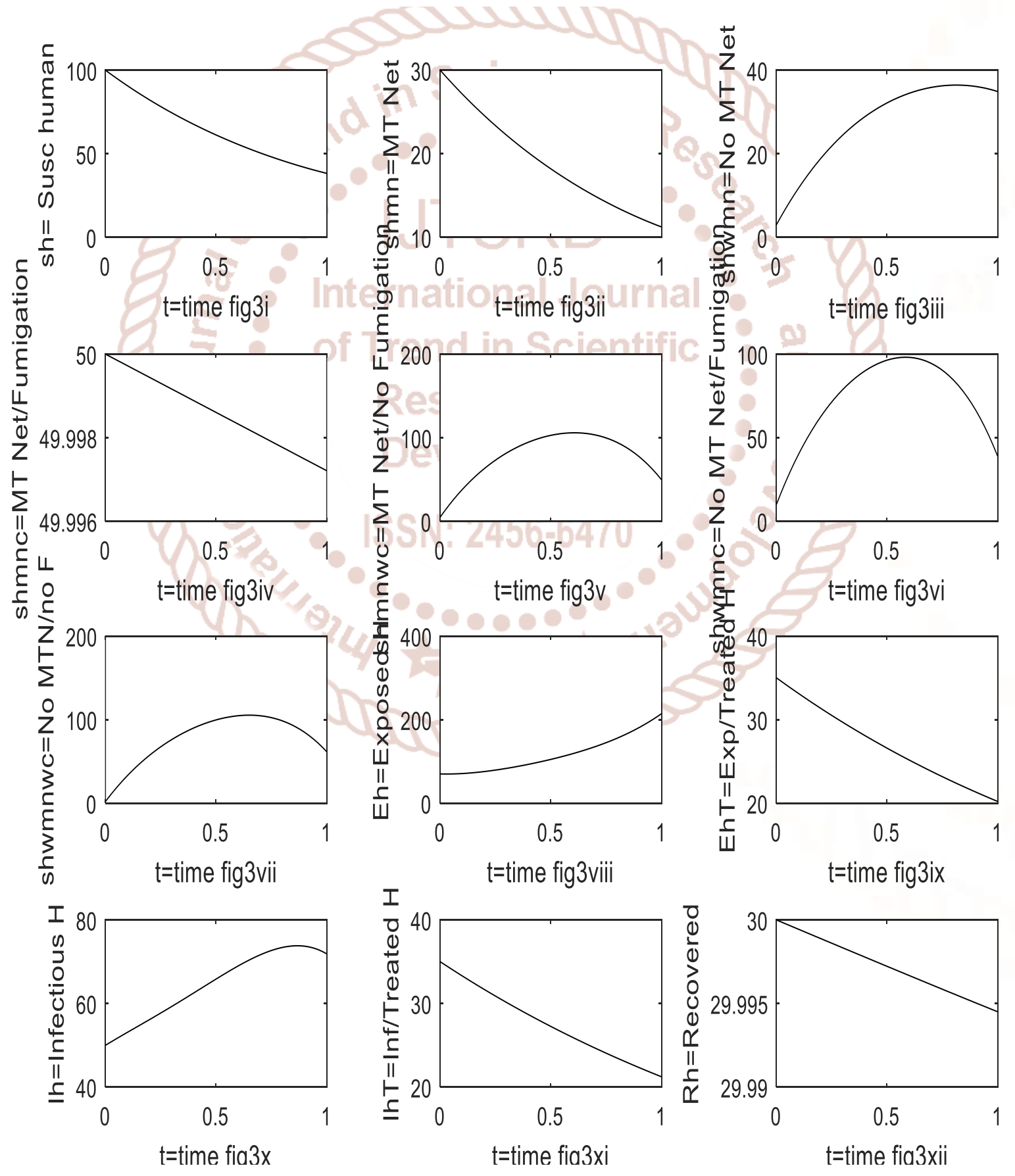


International Journal of Trend in Scientific Research and Development (IJTSRD) ISSN: 2456-6470
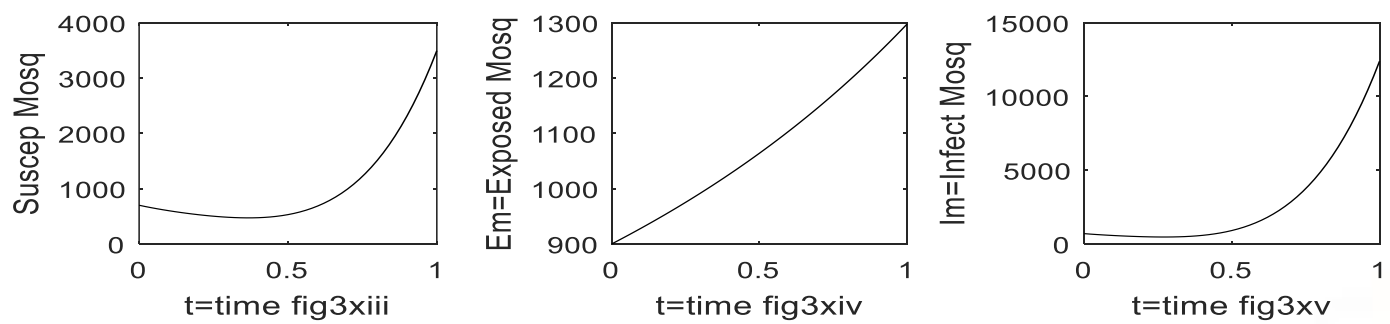

Figures (3i-3xv): Numerical Simulation of model system (3.1) when there is no
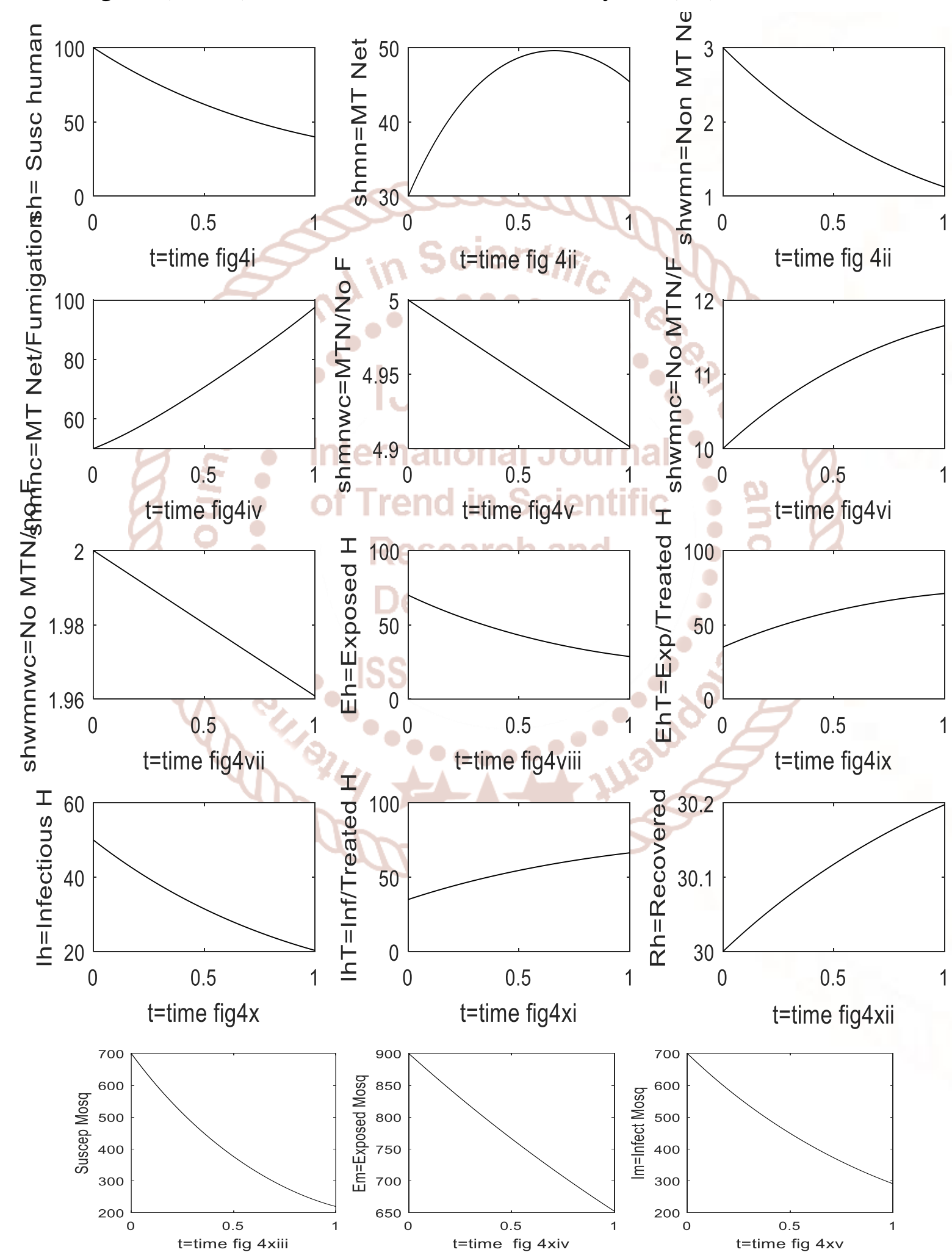

Figures (4i-4xv): Numerical Simulation of model system (3.1) when there is 
From figures $(2 \mathrm{a}-2 \mathrm{~b})$, we observe that the basic reproduction number which determines the dynamics of the disease in response to the control parameters is less than unity. This means that increase in $k$ and $r$ can slow disease spread. Eradication is easily done if $k$ and $r$ with the other control parameters is large relative to $k_{1}$ and $k_{2}$. Therefore, increasing $k$ and $r$ means increasing the effectiveness of the control measures, thus, malaria disease spread can be stopped.

Figures $(3 i-3 x v)$ shows the behavior of Susceptible human, Susceptible human who use mosquito net, Susceptible human who doesn't use mosquito net, Susceptible human who use mosquito net and fumigate their surroundings, Susceptible human who doesn't use mosquito net but fumigate their surroundings, Susceptible human who use mosquito net but doesn't fumigate their surroundings, Susceptible human who doesn't use mosquito net and doesn't fumigate their surroundings, Exposed (infected) human, Exposed (infected) but treated human, Infectious human, Infectious but treated human and Recovered human together with Susceptible mosquito, Exposed (infected) mosquito and Infectious mosquito when there is no control. We observe that the susceptible human population drops because the population of exposed (infected) and infectious human increases as the population of exposed (infected) and infectious mosquitoes increases.

Similarly, figures $(4 i-4 x v)$ depict the varying effects of the proportion of the control measures against mosquitoes and malaria infection. As shown in figures $(4 i-4 x v)$, the population of susceptible mosquitoes decrease with time as a result of the fact that the density of human who use insecticide treated bed nets and fumigate their surroundings increases. Also observed here is the increase in the density of the exposed but treated and infectious but treated humans and this indicates that the treatment is effective. We therefore state that when sufficient human population effectively use insecticide treated bed nets and adequately fumigate their environment, his will reduce the density of the exposed (infected) humans. More so, the application of an effective therapy will reduce the density of the infectious humans as indicated by the simulation.
In conclusion, the numerical simulation result showed that combination of the use of insecticide treated bed nets, fumigation of the surroundings and adequate treatment can effectively control malaria infection.

\section{Concluding Remarks}

This paper designed and analyzed a compartmental model for malaria transmission dynamics in human and mosquito population with combined control methods. The human population was divided into twelve compartments of Susceptible human, Susceptible human who use mosquito net, Susceptible human who doesn't use mosquito net, Susceptible human who use mosquito net and fumigate their surroundings, Susceptible human who doesn't use mosquito net but fumigate their surroundings, Susceptible human who use mosquito net but doesn't fumigate their surroundings, Susceptible human who doesn't use mosquito net and doesn't fumigate their surroundings, Exposed (infected) human, Exposed (infected) but treated human, Infectious human, Infectious but treated human and Recovered human while the mosquito population was divided into three compartments of Susceptible mosquito, Exposed (infected) mosquito and Infectious mosquito, since infectious mosquito does not recover from the infection.

We showed that the system is epidemiologically and mathematically well - posed by determining the positive invariant and the disease free equilibrium point was also shown. The basic reproduction number was obtained using the next generation matrix technique. We prove that the disease free equilibrium is asymptotically stable when the basic reproduction number is less than one and unstable when is greater than one.

Finally we numerically simulate our model to see the effect of the control parameters. Our result showed that increasing the proportion of humans who use insecticide treated bed net and fumigate their surroundings will go a long way in reducing human mosquito contact rate, thereby reducing the density of the exposed (infected) human in the population. Similarly, effective treatment of infectious human helps to boost their immunity as this will prevent the occurrence of malaria infection over time. Therefore, combined control is more effective than when there is a single control method or when there no control. 
International Journal of Trend in Scientific Research and Development (IJTSRD) ISSN: 2456-6470

\section{References}

1. Agusto $\mathrm{F} \mathrm{B}$, Del Valle $\mathrm{S} \mathrm{Y}$, Blayneh $\mathrm{K} \mathrm{W}$, Ngonghala C N, Gonkalves M J, Li N, Zhao R and Gong H. (2013). The Impact of Bed Net Use on Malaria Prevelence, . NIH-PAJ THeor. Biol.,320, 58-65.

2. Barnes K I, Durrheim D N, Little F et al. (2005). Effect of Artemether - Lumefantrine policy and improved vector controlon mnalaria burden i Kwazulu-Natal, South Africa. PLoS Med, 2:e330.

3. Beier J C, Keating J, Githure J I, Macdonald M B, Impoinvil D E and Novak R J. (2008). Integrated Vector Management for Malaria Control. Malar $J ;$; 7(supp): S4.

4. Birkhoff G and Roff G C (1989) ordinary differential equation, John Wiley and sons, New York, NY, USA, USA $4^{\text {th }}$ edition.

5. Buonomo B. (2013). Analysis of aMalaria Model with mosquito host choice and Bed Net Control,. University of Naples Federico II Naples, , arXiv: 1311.3209.

6. Chinebu T I, Ezennorom E O and Mbah G C E. (2018). A mathematical model of the Dynamicsof Hepatitis B Virus (HBV) infection and Cotrols. International Journal of Trend in Scientific Research and Development (IJTSRD, Vol.2, Issue 5, pp. 1654-1677 ; ISSN NO. 2456-6470.

7. Chitnis N, Cushing J M, and Hyman J M. (2006). Bifurcation analysis for a mathematical model for malaria Transmission. SIAM J. APPL. MATH, Society For Industrial and Applied Mathematics, vol 67.

8. Chitnis N, Schapira A, Smith T and Steketee R. (83(2010)). Comparing the Effectiveness of malaria Vector - Control Interventions through a Mathematical Model. Am.J.Trop Med.Hyg,, 230240.

9. Chiyaka C, Garira W and Dube S. (2007). Transmission model of Endemic Human Malaria in partially immune population. Elsevier, Mathematical computer modelling.

10. Chiyaka C, Garira W and Dube S. (2008). Modelling Immune Response and Drug Therapy in Human Malaria Infection. Computational and Mathematical method in Medicine, Vol.9, No. 2, 143-163.

11. Diekmann O and Heesterbeek J A P (1990). On the definition and conputation of basic reproduction number Ro in models for infectious diseases in heterogeneous populations. J. Math. Biol. , 28, 365-382.

12. Dietz K L and Molinoaux A T. (1974). A Malaria Model Tested in Africa Savannah. Bull, WHO

13. El-Nor. Osman M A and Adu I K. (2017). Simple mathematical model for malaria Transmission. Journal of Advances in mathematics and computer Science (JAMCS.37843), 25(6): 1-24.

14. Enayati A and Hamingway J. (2010). Malaria Management Past, Present and Future. Annu Rev Entomol, 55: 569-91.

15. Godfray H C. (2013). Mosquito Ecology and control of Malaria. J. Anim Ecol;, 82: 15-25.

16. Hawlay W A, ter Kuile F O, Steketee R S, Nahlen B L, Terlouw, D J, Gimnig J E, Shi Y P, Vulule J M, Alaii J A, Hightower A W, Kolczak M S, Kariuki S K, and Phillips-Howard P A. (2003). Implications of the westernKenya permethrintreated bed net study for policy, program implimentation, and Future research. American. Journal of Trop. Med. and Hygiene, 68, 168-173.

17. Kar T K and Jana S. (2013). A theoretical study on mathematical modeling of an infectious Disease with application of optimal control. Biosystems, vol. 111.no. 1, pp.37-50.

18. Liehl P, Meireles P, Albuquerque I S, Pinkevych M, Baptista F, Mota MM, Davenport MP, Prudencio M. (2015). Innate Immunity induced by Plasmodium liver infection inhibits malaria reinfections. Infect Immune, 83:11721180doi:10.1128/IAI.02796-14.

19. Macdonald G. (1957). The Epidemiology and Control of Malaria. Oxford: Oxford University Press.

20. Moonen B, Cohen J M, Snow R W et al. (2010). Operational Strategies to achieve and maintain malaria elimination. Lancet, 376;1592-6003.

21. Ngwa G A and Shu W S. (2000). A mathematical Model for the endemic malaria with variable human and mosquito populations. Math. Comput. Modelling, pp. 747-763.

22. Olaniyi S and Obabiyi O S. (2013). mathematical Model for Malaria Transmission Dynamics on Human and Mosquito population with nonlinear force of Infection. International Journal of Pure and Applied Mathematics , 88(1) : 125-150. 
23. Otieno G, Koske J K and Mutiso J M. (2016). Transmision Dynamics and Optimal Control of Malaria in Kenya. Discrete Dynamics in Nature and Society, Article ID8013574, 27 pages.

24. Padney R, Singh R N and Padney P N. (2013). Mathematical model for Malaria Transmission and Chemical Control. Journal of International Academy of Physical Sciences, vol.17, No. 4, PP 377-394. ISSN 0974-9373.

25. Phyo A P,Nkhomas S, Stepniewska K, Ashley E A, Nair, S, Mc Gready R, Al-Saai S, Dondorp A M, Lwin K M, Singhassivanon P et al. (2012). Emergence of artemisinin-Resistance Malaria on the western boarder of Thailand: Alongitudinalstudy. Lancet 379(9830), 19601966.

26. Pluess B, Tanser F C, Lengeler C and Sharp B L. (2010). Indoor residual spraying for prevention malaria. Cochrane Database Syst Rev., 4: CD006657.

27. Ross, R. (1910). The Prevention of Malaria. London: John Murray.

28. Tumwiine J, Mugish J Y T, Luboosi L S, . (2007). A mathematical model for the Dynamics of Malaria in a Human Host and Mosquito Vector with Temporary Immunity. Elisevier Applied Mathematical and Computation.

29. Van den Driessche P and Watmough J. (2002). Reproduction number and sub threshold endemic equilibria for compartmental models of disease transmission. Math. Biosci. , 180, 29-48.

30. World Health Organization. (2000-2010). Global Report on anti malarial Drug Efficacy and Drug Resistance. Tech- Rep, World Health Organization.

31. World HealthOrganization. (2004). Global Strategies Framework for integrated vector management. Geneva: World Health Organization.

32. World HealthOrganization. (2013). World Malaria Report. WHO Global Malaria Program, Geneva 2013.

33. World HealthOrganization. (2014). Vector Borne Diseases. WHOFact Sheet No 387, Geneva, 2014 , No.

34. Yang H M. (2000). Malaria transmission model for different Levels of acquired immunity and Temperature-dependent parameters (vector). Journal of Public Health, volume 34. 